\title{
FLEXINVERT: an atmospheric Bayesian inversion framework for determining surface fluxes of trace species using an optimized grid
}

\author{
R. L. Thompson and A. Stohl \\ Norwegian Institute for Air Research, Kjeller, Norway \\ Correspondence to: R. L. Thompson (rona.thompson@nilu.no)
}

Received: 20 May 2014 - Published in Geosci. Model Dev. Discuss.: 10 June 2014

Revised: 21 August 2014 - Accepted: 31 August 2014 - Published: 30 September 2014

\begin{abstract}
We present a new modular Bayesian inversion framework, called FLEXINVERT, for estimating the surface fluxes of atmospheric trace species. FLEXINVERT can be applied to determine the spatio-temporal flux distribution of any species for which the atmospheric loss (if any) can be described as a linear process and can be used on continental to regional and even local scales with little or no modification. The relationship between changes in atmospheric mixing ratios and fluxes (the so-called source-receptor relationship) is described by a Lagrangian Particle Dispersion Model (LPDM) run in a backwards-in-time mode. In this study, we use FLEXPART but any LPDM could be used. The framework determines the fluxes on a nested grid of variable resolution, which is optimized based on the source-receptor relationships for the given observation network. Background mixing ratios are determined by coupling FLEXPART to the output of a global Eulerian model (or alternatively, from the observations themselves) and are also optionally optimized in the inversion. Spatial and temporal error correlations in the fluxes are taken into account using a simple model of exponential decay with space and time and, additionally, the aggregation error from the variable grid is accounted for. To demonstrate the use of FLEXINVERT, we present one case study in which methane fluxes are estimated in Europe in 2011 and compare the results to those of an independent inversion ensemble.
\end{abstract}

\section{Introduction}

Observations of atmospheric mixing ratios (or concentrations) of trace species (gases or aerosols) contain information about their fluxes between land/ocean and the atmosphere.
Atmospheric inversions use this information formally in a statistical optimization to find spatio-temporal distributions of trace gas (or aerosol mass) fluxes (e.g. Tans et al., 1990). This can be done provided that there is a model of atmospheric transport relating changes in fluxes to changes in mixing ratios (or concentrations) - that is, the so-called source-receptor relationships (SRRs). Basically, two types of models are used: Eulerian models, in which atmospheric transport and chemistry are calculated relative to a fixed coordinate, or Lagrangian Particle Dispersion Models (LPDMs), in which diffusion and chemistry are calculated from the perspective of air parcels transported by ambient winds.

Eulerian models have been used extensively in atmospheric inversions but have a disadvantage in that SRRs cannot be calculated directly from the model. Instead, the SRRs can be found from multiple runs of the transport model to determine the sensitivity of all receptors to changes in the fluxes in a discrete number of regions (e.g. Fung et al., 1991; Enting, 2002; Rayner et al., 1999). This approach is simple to implement but the computational cost of running the model for each flux region limits the number of regions and thus the resolution that can be used. More recently, adjoints of Eulerian transport models have been developed and can be used as an alternative to calculating SRRs. Adjoints calculate the partial derivative of the change in flux in a given grid cell to the change in mixing ratio at a given point and time, which can be used to find the optimal fluxes based on a set of observations (e.g. Kaminski et al., 1999; Chevallier et al., 2005). This approach has the advantage that the fluxes can be solved at higher resolution (i.e. the resolution of the transport model). However, deriving adjoint models represents a significant technical challenge, thus adjoints are not available for all transport models and there is a significant lag between 
forward model developments and these being implemented in the adjoint. A further disadvantage is that these systems are computationally demanding, as they require forward and adjoint model runs for every iteration until convergence.

LPDMs are self-adjoint, i.e. they can track the dispersion of virtual particles representing e.g. an atmospheric gas forward in time from its sources/sinks to receptors (i.e. measurement sites) or backwards in time from receptors to its sources/sinks using the identical model formulation (Stohl et al., 2003; Seibert and Frank, 2004; Flesch et al., 1995). The forward and backward calculations are equivalent but one direction can be much more computationally efficient than the other. For instance, if there are few receptors but many sources/sinks, the backwards mode is more efficient. This is the case, for instance, when particles are tracked backwards from a relatively small number of available atmospheric observation sites (i.e. receptors), as in our demonstration case. This feature makes LPDMs very efficient for the purpose of atmospheric inversion and they have been previously used in numerous studies (e.g. Gerbig et al., 2003; Lauvaux et al., 2009; Stohl et al., 2010; Thompson et al., 2011; Keller et al., 2012; Brunner et al., 2012). Lagrangian models may be used on a global scale (e.g. Stohl et al., 2010), sub-continental scale (e.g. Gerbig et al., 2003) or on a regional scale of the order of a few hundred square kilometres (e.g. Lauvaux et al., 2009). Owing to their favourable treatment of atmospheric turbulence in the boundary layer, LPDMs can even be used down to scales of a few hundreds of metres (Flesch et al., 1995) and have been used for inferring source strengths for local sources (e.g. farmsteads and oil spills). A further advantage of LPDMs is that they can be run backward exactly from a measurement site, unlike Eulerian models, in which site measurements are represented by the averaged value of the corresponding grid cell. By focusing on local or regional scales, fine resolution may be used without running into problems of too large a number of unknown variables (in this case the fluxes). Fine resolution is desirable as it reduces the model representation error, also known as aggregation error (Kaminski et al., 2001; Trampert and Snieder, 1996) but it must be traded-off with the total number of flux variables to be determined, which is subject to computational constraints.

Using LPDMs to solve the inverse problem, however, also has disadvantages. In LPDMs, virtual particles are typically followed backward in time only for the order of days to a few weeks, thus the influence of the atmospheric chemistry and transport and surface fluxes further back in time (the socalled background mixing ratio) must be taken into account separately. Although forward 3-D simulations in LPDMs are possible, in order to reproduce background signals, such as seasonal variability, simulations of months to years would be necessary and, therefore, computationally too expensive (Stohl et al., 2009). Alternatively, the background mixing ratio can be accounted for using either observation- or modelbased approaches. Observation-based approaches use some filtering method (either statistical or based on meteorological criteria) to identify observations representative of the background, i.e. air not (or only minimally) influenced by fluxes during the time of the backwards calculations (e.g. Stohl et al., 2009; Manning et al., 2011). However, the background is strongly influenced by meteorology - e.g. air transported from higher latitudes or altitudes may have significantly different mixing ratios compared to air transported from lower latitudes or altitudes even if in both cases no emissions occur during the backward calculation. This makes the determination of an observation-based background difficult. Modelbased approaches involve coupling the back-trajectories at their point of termination to the mixing ratios determined from a global model.

One approach is to run the LPDM on a regional domain and couple this to a global model at the domain boundary. This approach was adopted by Rödenbeck et al. (2009), who use a two-step method to first solve for the fluxes on a coarse grid using an Eulerian model and to calculate the background mixing ratios at the receptors, and second to perform the inversion at regional scale on a finer grid using an LPDM. A similar approach was developed by Rigby et al. (2011) but using a one-step method. A drawback of both these approaches, however, is that only the coarse-resolution Eulerian model is used to calculate the background mixing ratios at the receptors and, thus, is more susceptible to transport errors. We use a different approach and couple the LPDM, run on a global scale, to an Eulerian model at the time boundary, such as done by Koyama et al. (2011). This approach utilizes the more accurate transport of the LPDM to calculate the background at the receptors.

In this paper, we present a new framework, called FLEXINVERT, for optimizing fluxes by employing an LPDM that can be coupled to mixing ratio fields from a global (Eulerian) model. This method may be used from large continental scales down to local scales and can be used for sparse as well as dense observation networks. In this method, the LPDM is used to transport air masses and, thus, the influence of fluxes, to each receptor. The fluxes inside the domain are optimized on a grid of variable resolution, where finer resolution is used in areas with a strong observation constraint, i.e. close to receptors, and coarser resolution is used elsewhere. FLEXINVERT, as it is presented here, requires that the LPDM is run on a global domain, or at least that the domain is large enough so that trajectories do not exit the domain. In summary, the features of this method are:

- atmospheric transport (SRR) is calculated using a single model, i.e. the LPDM;

- the LPDM needs only to be run once for each species and receptor to find the SRRs, as the output can be applied to optimize the fluxes for any domain and resolution (as long as the resolution is no finer than that of the LPDM run); 
- the variable resolution grid means that fine resolution close to receptors minimizes model representation errors;

- background mixing ratios can be provided either by coupling to mixing ratio output from a global model or alternatively by using an observation-based method;

- the background mixing ratios are optionally included in the optimization;

- the influence of fluxes from outside the domain on the mixing ratios at the receptors is accounted for without having to solve for them explicitly, thereby reducing the dimensionality of the problem.

Variable grid resolution has been used in atmospheric inversions previously such as in the studies of Manning et al. (2003), Stohl et al. (2010) and Wu et al. (2011). Our method for defining the variable grid is based on that of Stohl et al. (2010). However, we have also implemented a re-optimization of the fluxes at variable resolution back to the finest model resolution based on the method of $\mathrm{Wu}$ et al. (2011).

This paper is structured as follows: first we describe the inversion framework and the variable grid formulation and, second, we present an example using real observations of methane $\left(\mathrm{CH}_{4}\right)$ dry-air mole fractions to optimize $\mathrm{CH}_{4}$ emissions in Europe.

\section{Bayesian framework for linear inverse problems}

\subsection{Forward model}

For cases where the atmospheric transport and chemistry are linear, the change in mixing ratio of a given atmospheric species can be related to its fluxes by a matrix operator. Furthermore, the absolute mixing ratio can be related to its fluxes plus the background mixing ratio, which together form the so-called state vector. This is shown in Eq. (1) where $\boldsymbol{y}_{(M \times 1)}^{\mathrm{mod}}$ is a vector of the modelled mixing ratio at $M$ points in time and space, $\boldsymbol{x}_{(N \times 1)}$ is a vector of the $N$ state variables discretized in time and space, and $\mathbf{H}_{(M \times N)}$ is the transport operator:

$\boldsymbol{y}^{\mathrm{mod}}=\mathbf{H} \boldsymbol{x}$.

For simplicity, we describe the case where the state variables are optimized for only one time step, although the framework is able to optimize many time steps simultaneously (for an overview of the variables and their dimensions see Tables 1 and 2). We construct the matrix $\mathbf{H}$ from three components of the atmospheric transport to each receptor: (1) transport of fluxes within a nested domain (i.e. within the global domain), $\mathbf{H}^{\text {nest }}$, (2) transport of fluxes outside the nested domain, $\mathbf{H}^{\text {out }}$, and (3) contribution of mixing ratios at

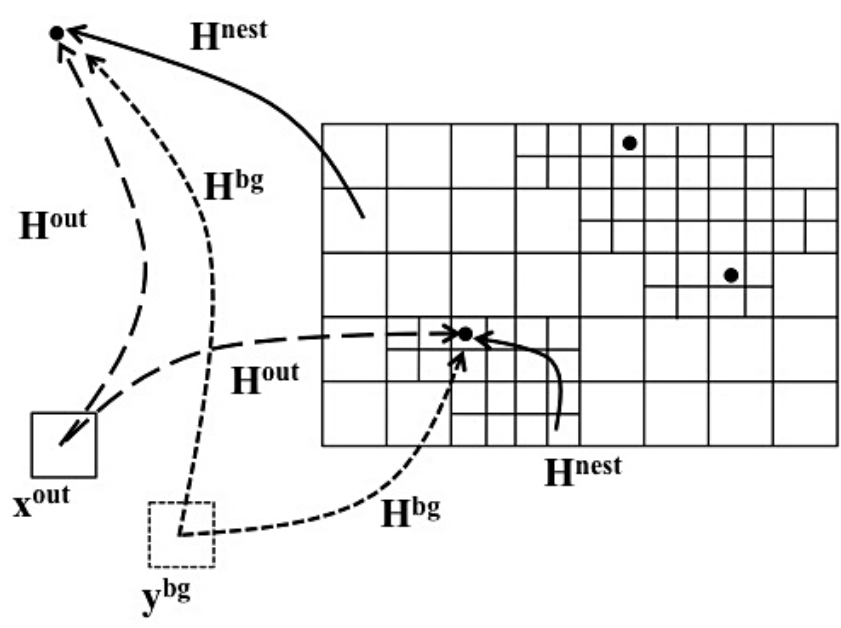

Figure 1. Schematic showing how the forward model is defined. The black dots represent receptors, the solid boxes represent gridded fluxes and the dotted box represents gridded mixing ratios from global model output. The arrows indicate transport to a receptor (which may be either inside or outside the nested domain): solid arrows show transport from fluxes within the nested domain, dashed arrows indicate transport from fluxes outside the nested domain, and the dotted arrows indicate transport of the mixing ratio at the point of back-trajectory termination. Each arrow can be thought of as an element (i.e. a partial derivative) in the transport matrix, $\mathbf{H}^{\text {nest }}$, $\mathbf{H}^{\text {out }}$, and $\mathbf{H}^{\text {bg }}$, respectively.

the time and location when the back-trajectories terminate, i.e. the initial mixing ratios, $\mathbf{H}^{\text {bg }}$ (see Fig. 1). Similarly, $\boldsymbol{x}$ is constructed from the fluxes inside the domain, $f^{\text {nest }}$, fluxes outside the domain, $f^{\text {out }}$ (there are no common variables between $f^{\text {nest }}$ and $f^{\text {out }}$ so there is no double counting of fluxes), and initial mixing ratios taken from the output of a global model, $\boldsymbol{y}^{\mathrm{bg}}$. (Note that here we refer to the contribution to the observed mixing ratio from where the trajectories terminate as the initial mixing ratio and the contribution from the initial mixing ratio plus from the fluxes outside the domain as the background mixing ratio - this is explained in Sect. 2.1.2). Equation (1) can thus be expanded to

$\boldsymbol{y}^{\text {mod }}=\mathbf{H}^{\text {nest }} \boldsymbol{f}^{\text {nest }}+\mathbf{H}^{\text {out }} \boldsymbol{f}^{\text {out }}+\mathbf{H}^{\text {bg }} \boldsymbol{y}^{\text {bg }}$.

\subsubsection{The source-receptor relationships (SRRs)}

The matrices $\mathbf{H}^{\text {nest }}$ and $\mathbf{H}^{\text {out }}$ are Jacobians in which each element is a partial derivative of the change in mixing ratio at a given receptor with respect to the change in mass flux in a given grid cell and are built from the SRRs. In this study, we use the LPDM, FLEXPART (Stohl et al., 2005, 1998) to derive the SRRs, although any other LPDM capable of running in backwards mode could also be used to construct these matrices.

In an LPDM, ensembles of particles are released from each receptor point and their displacement is calculated backwards in time based on wind fields from meteorological 
Table 1. Overview of the variables used in this manuscript

\begin{tabular}{|c|c|c|}
\hline Variable & Dimension & Description \\
\hline$y^{\bmod }$ & $M \times 1$ & modelled atmospheric mixing ratios \\
\hline$x$ & $N \times 1$ & state vector \\
\hline $\mathbf{H}$ & $M \times N$ & complete atmospheric transport operator \\
\hline$f^{\text {nest }}$ & $K \times 1$ & fluxes inside the nested domain \\
\hline $\mathbf{H}^{\text {nest }}$ & $M \times K$ & atmospheric transport operator for inside the nested domain \\
\hline$f^{\text {out }}$ & $P \times 1$ & fluxes outside the nested domain \\
\hline $\mathbf{H}^{\text {out }}$ & $M \times P$ & atmospheric transport operator for outside the nested domain \\
\hline $\boldsymbol{y}^{\mathrm{bg}}$ & $P \times 1$ & mixing ratios from the global model interpolated to the fine grid globally \\
\hline $\mathbf{H}^{\mathrm{bg}}$ & $M \times P$ & sensitivity to initial mixing ratios from the global model \\
\hline$\Gamma$ & $W \times K$ & prolongation operator from the fine to the variable grid in the nested domain \\
\hline$f_{\mathrm{vg}}^{\text {nest }}$ & $W \times 1$ & fluxes inside the nested domain on the variable grid \\
\hline $\mathbf{H}_{\mathrm{vg}}^{\text {nest }}$ & $M \times W$ & atmospheric transport operator for fluxes on the variable grid \\
\hline $\mathbf{M}_{\mathrm{cg}}^{0}$ & $M \times R$ & total background mixing ratios \\
\hline$\Gamma_{\mathrm{cg}}$ & $R \times P$ & prolongation operator from fine to coarse grid \\
\hline$\Gamma_{\mathrm{bg}}$ & $R \times M$ & prolongation operator for observation-based background mixing ratios \\
\hline $\boldsymbol{a}_{\mathrm{cg}}$ & $R \times 1$ & background mixing ratio scalars \\
\hline$x_{\mathrm{b}}$ & $N \times 1$ & prior state vector of fluxes and background mixing ratio scalars \\
\hline$\sigma$ & $W \times 1$ & prior flux error vector \\
\hline B & $N \times N$ & prior error covariance matrix on the variable grid \\
\hline $\mathbf{B}_{\mathrm{vg}}^{\text {flux }}$ & $W \times W$ & prior error covariance matrix for the fluxes on the variable grid \\
\hline $\mathbf{B}^{\text {flux }}$ & $K \times K$ & prior error covariance matrix for fluxes on the fine grid \\
\hline $\mathbf{R}$ & $M \times M$ & observation error covariance matrix \\
\hline $\mathbf{C}$ & $W \times W$ & spatial and temporal correlation matrix \\
\hline $\mathbf{C}_{\mathrm{S}}$ & $W \times W$ & spatial correlation matrix \\
\hline $\mathbf{C}_{\mathrm{T}}$ & $1 \times 1$ & temporal correlation matrix (trivial case when only one time step is used) \\
\hline A & $N \times N$ & posterior error covariance matrix \\
\hline $\mathbf{A}^{\text {flux }}$ & $W \times W$ & posterior error covariance matrix for fluxes on the variable grid \\
\hline$f^{\text {nest* }}$ & $K \times 1$ & posterior fluxes optimized on the fine grid \\
\hline $\mathbf{A}^{\text {flux* }}$ & $N \times N$ & posterior error covariance matrix for fluxes on the fine grid \\
\hline $\mathbf{P}$ & $Q \times K$ & operator to select variables that violate the inequality constraint \\
\hline$c$ & $Q$ & a vector of inequality constraints \\
\hline
\end{tabular}

analysis data. Backwards and forwards calculations are practically equivalent because the transport is time reversible (Seibert and Frank, 2004). For a tracer, which undergoes negligible loss in the atmosphere on the timescale of the LPDM calculations, the SRR can be expressed for a receptor and a flux in a given spatio-temporal grid cell $(i, n)$, as proportional to the average residence time of $J$ back-trajectories in the grid cell under consideration:

$$
\frac{\partial y}{\partial x_{i, n}}=\frac{1}{J} \sum_{j=1}^{J} \frac{\Delta t_{i, j, n}^{\prime}}{\rho_{j, n}},
$$

where $\rho_{i, n}$ is the air density in the grid cell and $\Delta t_{i, j, n}^{\prime}$ is the residence time of trajectory $j$ in the spatio-temporal grid cell $(i, n)$ (see Seibert and Frank, 2004). In Eq. (3), the SRR is in units of residence time $\times$ volume per unit mass, which is integrated over the height of the surface layer in FLEXPART to be comparable with the surface flux, which is given per unit area. Atmospheric loss processes, such as reaction with the hydroxyl radical, dry and wet deposition, or radioactive decay, can be considered by including a transmission function in the right-hand side of Eq. (3), which quantifies the loss. The SRRs for all receptors and fluxes inside the nested domain constitute the elements of $\mathbf{H}^{\text {nest }}$ while the SRRs for all receptors and fluxes outside the domain constitute the elements of $\mathbf{H}^{\text {out }}$.

\subsubsection{Initial mixing ratios}

The initial mixing ratio is the contribution from the mixing ratios where the LPDM back-trajectories terminate. We include two alternatives for the calculation of the initial mixing ratio. The first alternative uses the sensitivity to the mixing ratio at the points in space and time where the LPDM back-trajectories terminate (calculated in FLEXPART) and the mixing ratio at those points taken from the output of a global model. The sensitivity to mixing ratio in a grid cell at a given time $(i)$ is calculated as the number of particle trajectories that terminate in the grid cell $\left(n_{i}\right)$ divided by the total number of particle trajectories released $(J)$ : 
Table 2. Overview of the dimension notation and their values in the case study (test S1).

\begin{tabular}{llr}
\hline Dimension & Description & Value \\
\hline$M$ & total number of observations & 1602 \\
$N$ & total number of state variables (12 time steps) & 13896 \\
$K$ & number of fine resolution grid cells in one time step & 2400 \\
$W$ & number of variable resolution grid cells in one time step & 1158 \\
$P$ & number of grid cells in global domain in one time step & 2700 \\
$R$ & number of coarse grid cells for the background mixing ratio & 144 \\
\hline
\end{tabular}

$\frac{\partial y}{\partial y_{i}}=\frac{n_{i}}{J}$

Again, loss processes can be considered by including a transmission function in the right-hand side of Eq. (4). The sensitivity to the mixing ratio in all $P$ grid cells (over the global domain) and for all $M$ observations is represented by the matrix $\mathbf{H}_{(M \times P)}^{\mathrm{bg}}$ and the mixing ratios from the global model by the vector $\boldsymbol{y}_{(P \times 1)}^{\mathrm{bg}}$, which has been interpolated to the resolution of the LPDM output. Thus, the initial mixing ratio at all receptors is $\mathbf{H}^{\mathrm{bg}} \boldsymbol{y}^{\mathrm{bg}}$.

The second alternative approximates the background mixing ratios from the observations themselves. In this case, the background mixing ratio is calculated in one step, i.e. there is no separate calculation of the initial mixing ratio and contribution from outside the nested domain. We have implemented a simple method involving selecting the lower quartile of the observations in a moving time window (e.g. 30 days) over the whole time series. This method was chosen as it is robust to the number of observations (i.e. it can be used for in situ as well as discrete measurements) although other more sophisticated background selection algorithms exist and could be used instead (e.g. Ruckstuhl et al., 2012; Giostra et al., 2011). The selected observations are the approximation for the contribution to the mixing ratio without any influence from fluxes in the nested domain thus, the corresponding elements of the prior modelled mixing ratio, $\mathbf{H}^{\text {nest }} \boldsymbol{f}^{\text {nest }}$, should be zero. However, this is not always the case, therefore, we also subtract the prior simulated mixing ratio from the selected observations so that there is zero contribution from fluxes inside the domain in the background mixing ratio. To avoid overestimating the contribution from inside the domain and, hence, underestimating the background mixing ratio, we also select the lower quartile of the prior simulated mixing ratios in a moving time window. Lastly, we calculate a running average of the background mixing ratios using a time window of 90 days, which is then linearly interpolated to the timestamp of the observations. This is done for each receptor. Similar methods for the background calculation have been used previously for cases where no reliable global model estimate of the mixing ratio was available (e.g. Stohl et al., 2010).

\subsection{Variable resolution grid}

To reduce the number of variables in the inversion problem we aggregate grid cells where there is little constraint from the atmospheric observations. In this way, we define a new vector of the fluxes to be optimized, $f_{\mathrm{vg}}^{\text {nest }}$, and transport matrix, $\mathbf{H}_{\mathrm{vg}}^{\text {nest }}$, which are on a grid of variable resolution ( $\mathrm{vg}=$ variable grid). The aggregation algorithm is based on time-averaged SRRs optionally convolved with the prior flux estimate. The variable grid is set up starting with a coarse grid, which is refined in a specified number of steps following the method of Stohl et al. (2009). For example, starting with a coarse resolution of $4^{\circ} \times 4^{\circ}$ the grid may be refined in two steps to resolutions of $2^{\circ} \times 2^{\circ}$ and $1^{\circ} \times 1^{\circ}$. The refinement is made so that the flux sensitivity (optionally multiplied by the prior flux) in each grid cell at its final resolution (e.g. $1^{\circ} \times 1^{\circ}$, $2^{\circ} \times 2^{\circ}$ and $4^{\circ} \times 4^{\circ}$ ) is above a given threshold. It is also optional whether or not to make the grid refinement over water bodies and ice so that grid cells with a water/ice area of $99 \%$ or more are not refined further, reflecting cases where the water/ice surface fluxes are either smaller, more homogeneous and/or more certain than the land surface fluxes. For determining which grid cells are land/water/ice we use the International Geosphere Biosphere Programme land-cover data set (IGBP-DIS) (Belward et al., 1999).

To convert from the fine to the variable grid, we define a projection operator $\Gamma_{(W \times K)}$ where $K$ is the number of grid cells in the nested domain at the original resolution and $W$ is the number at variable resolution. Each row of $\Gamma$ corresponds to a cell in the variable grid, and is a summation vector on the fine grid. The row vectors $\lambda_{i}$ of $\Gamma$ are orthogonal, thus $\lambda_{i} \lambda_{j}^{\mathrm{T}}=0$ for $i \neq j$ (since each fine grid cell can only belong to one variable grid cell). The flux vector $f^{\text {nest }}$ and the matrix $\mathbf{H}^{\text {nest }}$ on the variable grid can be found according to

$\boldsymbol{f}_{\mathrm{vg}}^{\text {nest }}=\Gamma \boldsymbol{f}^{\text {nest }} \quad$ and $\quad \mathbf{H}_{\mathrm{vg}}^{\text {nest }}=\mathbf{H}^{\text {nest }} \Gamma^{\mathrm{T}}$,

where "T" indicates the matrix transpose. The fluxes in $f$ nest are weighted by the ratio of the area of fine grid to the variable grid into which it is aggregated. The forward model on the variable resolution grid can thus be written as

$$
\boldsymbol{y}^{\mathrm{mod}}=\mathbf{H}_{\mathrm{vg}}^{\text {nest }} \boldsymbol{f}_{\mathrm{vg}}^{\text {nest }}+\mathbf{H}^{\text {out }} \boldsymbol{f}^{\text {out }}+\mathbf{H}^{\text {bg }} \boldsymbol{y}^{\mathrm{bg}}+\varepsilon^{\mathrm{agg}},
$$


where $\varepsilon_{(M \times 1)}^{\mathrm{agg}}$ is the model representation error from having reduced the resolution of the model (for a schematic for the forward model see Fig. 1). It is also known as the aggregation error and has been described by Trampert and Snieder (1996), Kaminski et al. (2001) and Thompson et al. (2011). We describe the calculation of the aggregation error in Sect. 2.5.

\subsection{Aggregation of the background mixing ratios}

The contribution of fluxes outside the domain to the change in mixing ratios at the receptor points (i.e. $\mathbf{H}^{\text {out }} \boldsymbol{f}^{\text {out }}$ ) can be added to the initial mixing ratio $\left(\mathbf{H}^{\mathrm{bg}} \boldsymbol{y}^{\mathrm{bg}}\right)$. The contribution to the modelled mixing ratio (i.e. $y^{\mathrm{mod}}$ ), which is not accounted for by the SRRs and fluxes inside the domain (i.e. the background mixing ratio), is then defined by a new matrix, $\mathbf{M}_{\mathrm{cg}(M \times R)}$, on a coarse grid (cg), which has rows corresponding to $M$ observations and columns corresponding to $R$ grid cells or latitudinal bands. When the initial mixing ratio is calculated using the sensitivity matrix, $\mathbf{H}^{\text {bg }}$ and mixing ratio fields, $\boldsymbol{y}^{\text {bg }}$ from a global Eulerian transport model, then $\mathbf{M}_{\mathrm{cg}}$ is defined as

$\mathbf{M}_{\mathrm{cg}}=\left(\mathbf{H}^{\text {out }} \circ \mathbf{F}^{\text {out }}+\mathbf{H}^{\mathrm{bg}} \circ \mathbf{Y}^{\mathrm{bg}}\right) \Gamma_{\mathrm{cg}}^{\mathrm{T}}$,

where $\circ$ indicates the Hadamard matrix product, $\mathbf{F}_{(M \times P)}^{\text {out }}$ has $M$ rows of $\left(f^{\text {out }}\right)^{\mathrm{T}}$, and $\mathbf{Y}_{(M \times P)}^{\mathrm{bg}}$ has $M$ rows of $\left(\boldsymbol{y}^{\mathrm{bg}}\right)^{\mathrm{T}}$. The matrix $\boldsymbol{\Gamma}_{\operatorname{cg}(R \times P)}$ is a projection operator from the Eulerian model resolution to a coarse resolution of $R$ grid cells (note $\Gamma_{\mathrm{cg}} \neq \Gamma$ ). Noteworthy, is that the matrix multiplication $\mathbf{H}^{\text {out }} \circ \mathbf{F}^{\text {out }}$ is made using the original resolution of the LPDM and fluxes and that the conversion to the coarse grid is performed only on the mixing ratios, thus avoiding an aggregation error in this component. When the background is calculated using the observations themselves, then $\mathbf{M}_{\mathrm{cg}}$ is defined as

$\mathbf{M}_{\mathrm{cg}}=\operatorname{diag}\left(\boldsymbol{y}^{\mathrm{bg}}\right) \Gamma_{\mathrm{bg}}^{\mathrm{T}}$,

where $\Gamma_{\mathrm{bg}(R \times M)}$ is an operator to map the background mixing ratios to a matrix where the background for each measurement is allocated to one of $R$ latitudinal bands. Note that the contribution from grid cells outside the domain is not explicitly included as it is assumed that this contribution is incorporated into the definition of $\boldsymbol{y}^{\text {bg }}$ when it is calculated from the observations (see Sect. 2.1.2).

For both methods, the columns of $\mathbf{M}_{\mathrm{cg}}$ correspond to the mixing ratios in each of the $R$ coarse-grid cells (or latitudinal bands when using the observation-based method) such that the sum of each row gives the total background mixing ratio for each measurement (note that for the observationbased method there is only one non-zero entry in each row). The spatial distribution of the contribution to the background mixing ratio (dimension $R$ ) is maintained as it is these contributions that are optimized in the inversion.
We then define a new transport operator $\mathbf{H}_{(M \times N)}$ by concatenating the matrices $\mathbf{H}_{\mathrm{vg}}^{\text {nest }}$ and $\mathbf{M}_{\mathrm{cg}}$. Similarly, we define the state vector $\boldsymbol{x}_{(N \times 1)}$ by concatenating $\boldsymbol{f}_{\mathrm{vg}(W \times 1)}^{\text {nest }}$ (the flux variables inside the nested domain) and $\mathbf{a}_{\mathrm{cg}}(R \times 1)$ (scalars of the mixing ratios in the columns of $\mathbf{M}_{\mathrm{cg}}$ ):

$\mathbf{H}=\left[\mathbf{H}_{\mathrm{vg}}^{\text {nest }} \mathbf{M}_{\mathrm{cg}}\right]$ and $\boldsymbol{x}=\left[\boldsymbol{f}_{\mathrm{vg}}^{\text {nest }} \boldsymbol{a}_{\mathrm{cg}}\right]$.

The prior value of $\boldsymbol{a}_{\mathrm{cg}}(i)(i=1$ to $R)$ is 1 . After inversion, the optimized values of $\boldsymbol{a}_{\mathrm{cg}}$ determine the posterior background mixing ratios.

\subsection{Optimization of the fluxes and background mixing ratios}

The uncertainty in the initial mixing ratios and in the contribution to the mixing ratio from fluxes outside the domain can be considerable. Therefore, we include this component in the optimization problem. The prior state vector for optimization, $\boldsymbol{x}_{\mathrm{b}}$, thus contains variables for the surface fluxes (on the variable-resolution grid) and variables for the optimization of the mixing ratios (on the coarse-resolution grid defined by $\Gamma_{\mathrm{cg}}$ ).

Based on Bayes' theorem, the most probable solution for $\boldsymbol{x}$ is the one that minimizes the difference between the observed and modelled mixing ratios while also depending on the prior state variables, $\boldsymbol{x}_{\mathrm{b}}$, and their uncertainties (for details on Bayes' theorem see e.g. Tarantola, 2005). Assuming that the uncertainties have a Gaussian probability density function (pdf) this can be described by the cost function

$$
\begin{aligned}
J(\boldsymbol{x})= & \frac{1}{2}\left(\boldsymbol{x}-\boldsymbol{x}_{\mathrm{b}}\right)^{\mathrm{T}} \mathbf{B}^{-1}\left(\boldsymbol{x}-\boldsymbol{x}_{\mathrm{b}}\right) \\
& +\frac{1}{2}\left(\mathbf{H} \boldsymbol{x}-\boldsymbol{y}^{\mathrm{obs}}\right)^{\mathrm{T}} \mathbf{R}^{-1}\left(\mathbf{H} \boldsymbol{x}-\boldsymbol{y}^{\mathrm{obs}}\right),
\end{aligned}
$$

where $\mathbf{B}_{(N \times N)}$ is the prior flux error covariance matrix (see Sect. 2.5), $\mathbf{R}_{(M \times M)}$ is the observation error covariance matrix (see Sect. 2.6), and $\boldsymbol{y}^{\mathrm{obs}}$ is a vector of the observed mixing ratios. There exist a number of methods to find the $\boldsymbol{x}$ for which Eq. (10) is at a minimum; we use the approach of finding the first derivative of Eq. (10) and solving this for $\boldsymbol{x}$. By rearrangement, $\boldsymbol{x}$ can be found according to Eq. (11). Equation (11) has a number of alternative formulations and the one we use is the most efficient when the number of observations is smaller than the number of unknowns, since the size of the matrix to invert $\left(\mathbf{H B H}^{\mathrm{T}}+\mathbf{R}\right)$ has dimensions of $M \times M$ :

$\boldsymbol{x}=\boldsymbol{x}_{\mathrm{b}}+\mathbf{B} \mathbf{H}^{\mathrm{T}}\left(\mathbf{H B H} \mathbf{H}^{\mathrm{T}}+\mathbf{R}\right)^{-1}\left(\boldsymbol{y}^{\mathrm{obs}}-\mathbf{H} \boldsymbol{x}_{\mathrm{b}}\right)$.

The inverse of $\left(\mathbf{H B H}^{\mathrm{T}}+\mathbf{R}\right)$ is found by Cholesky factorization (using the DPOTRF and DPOTRI routines from the LAPACK library). The corresponding posterior error covariance matrix, $\mathbf{A}_{(N \times N)}$, is the inverse of the second derivative of the cost function, $J$ :

$\mathbf{A}=\left(J^{\prime \prime}\right)^{-1}=\mathbf{B}-\mathbf{B} \mathbf{H}^{\mathrm{T}}\left(\mathbf{H B} \mathbf{H}^{\mathrm{T}}+\mathbf{R}\right)^{-1} \mathbf{H B}$. 


\subsection{Prior error covariance matrix}

Errors in the prior flux estimates are correlated in space and time owing to correlations in the biogeochemistry model, upscaling model, or anthropogenic emission inventory that was used to produce these estimates. Most often, there is little known about the true temporal and spatial error correlation patterns. Here we define the spatial error correlation for the fluxes as an exponential decay over distance, such that each element in the spatial correlation matrix $\mathbf{C}_{\mathrm{S}}$ is

$c_{\mathrm{S}(i, j)}=\exp \left(-\frac{d_{i j}}{k_{\mathrm{S}}}\right)$,

where $d_{i j}$ is the distance between grid cells $i$ and $j$ in a given time step and $k_{\mathrm{S}}$ is the spatial correlation scale length on land or ocean (we assume that fluxes on land and ocean are not correlated with one another). The temporal error correlation matrix $\mathbf{C}_{\mathrm{T}}$ is described similarly using the time difference between grid cells in different time steps. The full temporal and spatial correlation matrix $\mathbf{C}$ is given by the Kronecker product: $\mathbf{C}_{\mathrm{T}} \otimes \mathbf{C}_{\mathrm{S}}$. The error covariance matrix for the fluxes, $\mathbf{B}_{\mathrm{vg}(W \times W)}^{\text {flux }}$, is the matrix product of correlation pattern, $\mathbf{C}$, and the error covariance of the prior fluxes, $\sigma \sigma^{\mathrm{T}}$, where $\sigma$ is a vector of the flux errors. We calculate the error on the flux in each grid cell (on the fine grid) as a fraction of the maximum value out of that grid cell and the eight surrounding ones. Finally, the $\mathbf{B}_{\mathrm{vg}}^{\text {flux }}$ matrix is scaled so that the square root of its sum is consistent with a total error value assigned for the whole domain. This error estimate may be from e.g. comparisons of independent biogeochemistry modelled fluxes or flux inventories. The correlation matrix could be calculated for the fine grid and converted to the variable grid using the prolongation operator as $\Gamma \mathbf{B}^{\text {flux }} \Gamma^{\mathrm{T}}$. However, we calculate $\mathbf{B}_{\mathrm{vg}}^{\text {flux }}$ directly for the variable grid (dimensions $W \times W)$ as the multiplication step $\mathbf{B}^{\text {flux }} \Gamma^{\mathrm{T}}$ is very slow if $K$ is large and/or if there are many time steps. In addition, $\mathbf{B}_{(K \times K)}^{\text {flux }}$ is calculated for the fine grid for a single time step only, as it is needed in the calculation of the aggregation error (see Sect. 2.6) and for the optimization of the posterior fluxes back to the fine grid (Sect. 2.8). We assume that the errors for the scalars of the background mixing ratios (i.e. $\boldsymbol{a}_{\mathrm{cg}}$ ) are uncorrelated and have a fixed prior value (e.g. $1 \%$ ). The error variance for these scalars is appended to $\mathbf{B}_{\mathrm{vg}}^{\text {flux }}$ to give $\mathbf{B}_{(N \times N)}$.

\subsection{Aggregation error}

The aggregation incurred by reducing the spatial resolution of the model can be calculated by projecting the loss of information in the state space into the observation space (Kaminski et al., 2001). The full aggregation error covariance matrix $\mathbf{E}_{(M \times M)}^{\mathrm{agg}}$ is given by

$\mathbf{E}^{\mathrm{agg}}=\mathbf{H} \Gamma_{-} \mathbf{B}^{\text {flux }} \Gamma_{-}^{\mathrm{T}} \mathbf{H}^{\mathrm{T}}$, where $\Gamma_{-}$is the projection of the loss of information in the variable grid compared to the fine grid. The matrix $\Gamma_{-}$can be calculated simply from the row vectors $\lambda_{i}$ of the projection operator $\Gamma$, which are weighted by the square root of the row sum so as to have unit length:

$\Gamma_{-}=\mathbf{I}-\sum_{i=1}^{W} \lambda_{i} \lambda_{i}^{\mathrm{T}}$,

where $\mathbf{I}$ is the identity matrix. As $\lambda_{i} \lambda_{i}^{\mathrm{T}}$ is a matrix of size $P \times P$, where $P$ can be on the order of 10000 to 100000 , it is not calculated directly but rather via $\mathbf{H} \Gamma_{\text {_ }}$ as follows:

$\mathbf{H} \Gamma_{-}=\mathbf{H}-\sum_{i=1}^{W} \mathbf{H} \lambda_{i} \lambda_{i}^{\mathrm{T}}$.

\subsection{Observation error covariance matrix}

The errors in the observation space incorporate measurement as well as model transport and representation errors. For the measurement errors, we use values of the measurement repeatability as given by the data providers. The measurement errors can be given as a single value or for each observation, in which case it is read from the observation files. Transport errors are extremely difficult to quantify and depend not only on the model but also on the input data, resolution and location. Therefore, we do not quantify the full transport error, but only the part of it that can be estimated from the model FLEXPART, i.e. the stochastic uncertainty, which arises by the representation of transport with a limited number of particles (see Stohl et al., 2005). The stochastic error, however, is likely to be much smaller than the full transport error. It is possible, however, to include an additional estimate of the transport error into Eq. (17), if this information were available. The error in the modelled mixing ratio is calculated using the stochastic uncertainty in the same way that the mixing ratios themselves are calculated. We consider two types of representation error: observation representation error and model aggregation error (discussed above). The observation representation error is calculated from the standard deviation of all measurements available in a user-specified measurement averaging time interval, based on the idea that if the measurements are fluctuating strongly within that interval then their mean value is associated with higher uncertainty than if the measurements are steady (e.g. Bergamaschi et al., 2010). If only one measurement is available during this interval, then a user-defined minimum error is used instead. The measurement and transport errors are assumed to be uncorrelated. Although this is a common assumption, correlations likely exist between e.g. hourly observations owing to errors in the modelled boundary layer height and wind fields, which could lead to temporal correlations. However, in the current version of FLEXINVERT, we do not account for these correlations. Hence, we define a diagonal matrix with elements equal to the quadratic sum of the measurement, transport 
model and measurement representation errors:

$\sigma^{2}=\sigma_{\text {meas }}^{2}+\sigma_{\text {trans }}^{2}+\sigma_{\text {repr }}^{2}$

Another assumption that is made is that the observedmodelled mixing ratio residuals have a Gaussian distribution (Eq. 10 is based on this assumption). Therefore, in cases where the distribution is highly skewed, observations corresponding to the tail of the distribution will have a strong influence on the result of the inversion. FLEXINVERT does not include any component to deal with skewed distributions; however, the influence of observations in the tail of the distribution may be reduced by increasing their uncertainty. For more details about dealing with skewed distributions we refer the reader to Stohl et al. (2009).

The observation error covariance matrix, $\mathbf{R}_{(M \times M)}$, is the sum of this diagonal matrix plus the aggregation error covariance matrix, $\mathbf{E}^{\mathrm{agg}}$.

\subsection{Optimization of the fluxes to fine resolution}

The optimal solution of the fluxes, $f_{\mathrm{vg}}^{\text {nest* }}$, is found for the variable grid according to Eq. (11) and the corresponding posterior error covariance matrix, $\mathbf{A}$, is found according to Eq. (12). However, it is not possible to directly apply the inverse of the projection operator to retrieve the optimal emissions at fine resolution since the operation from the variable to the fine resolution is ambiguous; there is insufficient information to redefine the fluxes at fine resolution. To find the optimal emissions at fine resolution, $f_{(K \times 1)}^{\text {nest* }}$, we use an adaptation of the method of Wu et al. (2011). This method involves a second Bayesian optimization step, which uses the prior information about the distribution of the fluxes within each grid cell on the variable resolution grid:

$$
\begin{aligned}
\boldsymbol{f}^{\text {nest* }}= & \boldsymbol{f}_{b}^{\text {nest }}+\mathbf{B}_{\text {naw }}^{\text {flux }} \Gamma_{\text {unit }}^{\mathrm{T}}\left(\Gamma \mathbf{B}^{\text {flux }} \Gamma^{\mathrm{T}}+\mathbf{A}^{\text {flux }}\right)^{-1} \\
& \left(\boldsymbol{f}_{\mathrm{vg}}^{\text {nest } *}-\Gamma \boldsymbol{f}_{b}^{\text {nest }}\right)
\end{aligned}
$$

(see Appendix A for the derivation of Eqs. 18 and 19). Since we only optimize the fluxes, i.e. $f^{\text {nest* }}$, the matrices $\mathbf{B}^{\text {flux }}$ and $\mathbf{A}^{\text {flux }}$ represent only the parts of the error covariance matrices corresponding to flux errors. We have introduced a new error covariance matrix, $\mathbf{B}_{\text {naw }}^{\text {flux }}$, which is the non-area-weighted (naw) version of $\mathbf{B}^{\text {flux }}$, i.e. calculated using the flux errors not weighted by the ratio of the grid cell areas on the fine and coarse grid. Also, we have introduced $\Gamma_{\text {unit }}$, which is equivalent to $\Gamma$ but with each row vector normalized by the row sum so that they have unit length. Our method departs from that of Wu et al. (2011) in that for the error in posterior state vector on the variable grid we use the error covariance of the posterior solution on the variable grid $\mathbf{A}$, rather than a Dirac distribution. The inverse of $\left(\Gamma \mathbf{B}^{\text {flux }} \Gamma^{\mathrm{T}}+\mathbf{A}^{\text {flux }}\right)$ is found by singular value decomposition (SVD) using the DGESDD routine from the LAPACK library. We find the posterior error
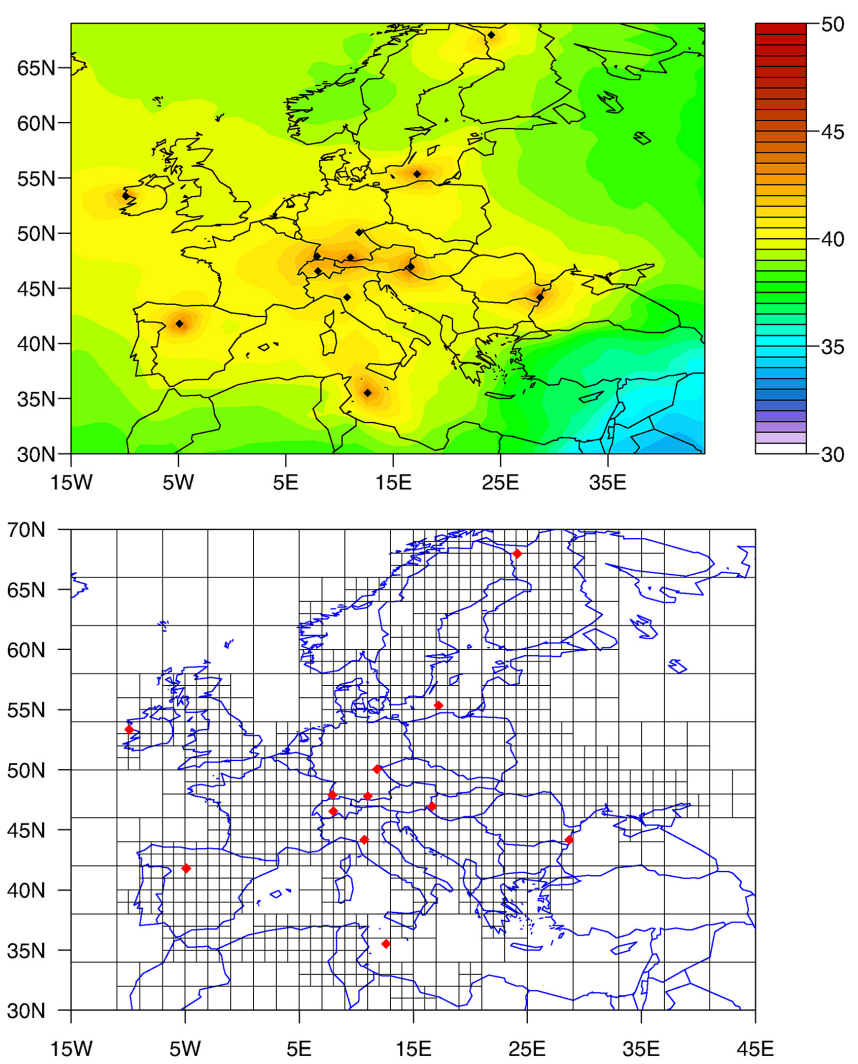

Figure 2. Total emission sensitivity for 2011 in units of $\log \left(\mathrm{s} \mathrm{m}^{-3} \mathrm{~kg}^{-1}\right)$ calculated using FLEXPART and used to determine the variable grid (note that for this no weighting is applied for the number of observations available from each site) (a) and the variable-resolution grid used in the inversion (b). The points indicate the positions of the observation sites.

covariance matrix $\mathbf{A}_{(K \times K)}^{\text {flux* }}$ also for the fine-resolution fluxes according to

$\mathbf{A}^{\text {flux* }}=\left(\mathbf{B}_{\text {naw }}^{\text {flux-1 }}+\Gamma_{\text {unit }}^{\mathrm{T}} \mathbf{A}^{\text {flux-1 }} \Gamma_{\text {unit }}\right)^{-1}$.

The inverse of the matrices $\mathbf{B}_{\text {naw }}^{\text {flux }}, \mathbf{A}^{\text {flux }}$, and $\left(\mathbf{B}_{\text {naw }}^{\text {flux-1 }}+\right.$ $\Gamma_{\text {unit }}^{\mathrm{T}} \mathbf{A}^{\text {flux-1 }} \Gamma_{\text {unit }}$ ) are also found by SVD, which can also be used for matrices that are non-positive definite. This optimization to the fine grid should be carefully evaluated if used. Alternatively, we also include a simple mapping back to the fine grid by distributing the flux in a coarse grid to the corresponding fine grid cells based on the prior relative flux distribution at fine resolution.

\subsection{Inequality constraints}

For some atmospheric species, there are physical restrictions on the values of the fluxes. For example, for anthropogenic species, such as halocarbons and $\mathrm{SF}_{6}$, the fluxes can only be positive over land, while there are no appreciable fluxes (positive or negative) over ocean. Using an inequality constraint 
Table 3. Atmospheric observation sites for $\mathrm{CH}_{4}$ mole fraction used in the case study. The altitude is given in metres above sea level.

\begin{tabular}{llrrrll}
\hline Site ID & Organization & Latitude & Longitude & Altitude $(\mathrm{m})$ & Type & Description \\
\hline PAL & FMI & $68.0^{\circ} \mathrm{N}$ & $24.1^{\circ} \mathrm{E}$ & 572 & $\mathrm{CM}$ & Pallas, Finland \\
ICE & NOAA & $63.3^{\circ} \mathrm{N}$ & $20.3^{\circ} \mathrm{W}$ & 127 & FM & Heimay, Vestmannaeyjar, Iceland \\
BAL & NOAA & $55.4^{\circ} \mathrm{N}$ & $17.2^{\circ} \mathrm{E}$ & 28 & FM & Baltic Sea, Poland \\
MHD & AGAGE & $53.3^{\circ} \mathrm{N}$ & $9.9^{\circ} \mathrm{W}$ & 40 & $\mathrm{CM}$ & Mace Head, Ireland \\
OXK & NOAA & $50.0^{\circ} \mathrm{N}$ & $11.8^{\circ} \mathrm{E}$ & 1185 & FM & Ochsenkopf, Germany \\
SSL & UBA & $47.9^{\circ} \mathrm{N}$ & $7.9^{\circ} \mathrm{E}$ & 1213 & $\mathrm{CM}$ & Schauinsland, Germany \\
HPB & NOAA & $47.8^{\circ} \mathrm{N}$ & $11.0^{\circ} \mathrm{E}$ & 990 & FM & Hohenpeissenberg, Germany \\
HUN & NOAA & $47.0^{\circ} \mathrm{N}$ & $16.7^{\circ} \mathrm{E}$ & 344 & FM & Hegyhatsal, Hungary \\
JFJ & EMPA & $46.6^{\circ} \mathrm{N}$ & $8.0^{\circ} \mathrm{E}$ & 3590 & $\mathrm{CM}$ & Jungfraujoch, Switzerland \\
PUY* & NOAA & $45.8^{\circ} \mathrm{N}$ & $3.0^{\circ} \mathrm{E}$ & 1475 & FM & Puy de Dôme, France \\
BSC & NOAA & $44.2^{\circ} \mathrm{N}$ & $28.7^{\circ} \mathrm{E}$ & 3 & FM & Black Sea, Constant, Romania \\
CMN & ISAC & $44.2^{\circ} \mathrm{N}$ & $10.7^{\circ} \mathrm{E}$ & 2165 & $\mathrm{CM}$ & Monte Cimone, Italy \\
CIB & NOAA & $41.8^{\circ} \mathrm{N}$ & $4.9^{\circ} \mathrm{W}$ & 845 & FM & CIBA, Spain \\
LMP & NOAA & $35.5^{\circ} \mathrm{N}$ & $12.6^{\circ} \mathrm{E}$ & 50 & FM & Lampedusa, Italy \\
\hline
\end{tabular}

* Only used for independent validation.

in the cost function Eq. (10) would mean that the first derivative would be undefined. Therefore, we adopt a "truncated Gaussian" approach following Thacker (2007), in which inequality constraints are applied by treating these as errorfree observations. The inequality constraints are applied to the posterior fluxes derived previously (i.e. with no inequality constraint). This is described by the following equation, which is analogous to Eq. (11):

$$
\begin{aligned}
f^{\text {nest } * *}= & f^{\text {nest } *} \\
& +\mathbf{A}^{\text {flux }} \mathbf{P}^{\mathrm{T}}\left(\mathbf{P} \mathbf{A}^{\text {flux }} \mathbf{P}^{\mathrm{T}}\right)^{-1}\left(\boldsymbol{c}-\mathbf{P} \boldsymbol{f}^{\text {nest } *}\right),
\end{aligned}
$$

where $\mathbf{P}_{(Q \times K)}$ is a matrix operator to select the $Q$ variables that violate the inequality constraint and $\boldsymbol{c}$ is a vector of the inequality constraints of length $Q$. The inequality constraint does not only affect the grid cells with negative values but there is also some adjustment to other cells according to the correlations described by the posterior error covariance matrix, $\mathbf{A}^{\text {flux }}$. The posterior error covariance matrix, however, is unchanged as the observation error covariance matrix in this case is zero. To apply the inequality constraint requires running a second code, which uses the output of FLEXINVERT.

A brief description of the software, its inputs and outputs, is provided in Appendix B.

\section{Case study: estimation of $\mathrm{CH}_{4}$ fluxes in Europe}

We provide a case study using FLEXINVERT for the estimation of methane $\left(\mathrm{CH}_{4}\right)$ fluxes in Europe. Methane was chosen, as it is an important greenhouse gas with an atmospheric lifetime of approximately 10 years (Denman et al., 2007) and since its loss in the troposphere is principally by reaction with the $\mathrm{OH}$ radical, which can be approximated as a linear process. The fluxes of $\mathrm{CH}_{4}$ are mostly positive (i.e. from the surface to the atmosphere) although small negative fluxes of $\mathrm{CH}_{4}$ by oxidation in soils are also possible (Ridgwell et al., 1999). Europe was chosen as it is reasonably well covered by observations, both discrete air sampling and in situ measurements. The important sources of $\mathrm{CH}_{4}$ in Europe are mostly anthropogenic - namely agriculture, landfills, and oil and gas exploitation (including fugitive emissions as well as those from incomplete combustion). Natural sources of $\mathrm{CH}_{4}$ are less important in Europe and principally wetlands and mostly in the higher latitudes. In this case study, we estimate the total fluxes of $\mathrm{CH}_{4}$ in the nested domain from 30 to $70^{\circ} \mathrm{N}$ and $15^{\circ} \mathrm{W}$ to $45^{\circ} \mathrm{E}$ at monthly resolution for the year 2011 .

\subsection{Inversion set-up}

\subsubsection{FLEXPART runs}

FLEXPART (version 8.1) (Stohl et al., 1998, 2005) was used to generate the SRRs by running 10-day backwards mode simulations from each of the receptors (i.e. the observation sites). FLEXPART was run at $1.0^{\circ} \times 1.0^{\circ}$ resolution with meteorological analyses from the European Centre for Medium-Range Weather Forecasts (ECMWF). Backwards ("retro-plume") simulations were made by releasing 20000 virtual particles in 3-hourly intervals and the SRRs (or equivalently emission sensitivities) were saved as $24 \mathrm{~h}$ averages. Particles were released from the sampling inlet height at each observation site (see Table 3). The loss of $\mathrm{CH}_{4}$ by reaction with the $\mathrm{OH}$ radical was also included in the backwards simulations even though the loss is very small over a 10-day period. Figure 2a shows the combined total emission sensitivity for all observation sites in 2011. The total emission sensitivity was used to determine the variable-resolution grid (Fig. 2b) with grid cells ranging in size from $1.0^{\circ} \times 1.0^{\circ}$ to $4.0^{\circ} \times 4.0^{\circ}$. 
Table 4. Methane flux estimates used in the prior in the case study.

\begin{tabular}{|c|c|c|}
\hline Source & Data set & $\begin{array}{l}\text { Total } \\
\left(\mathrm{TgCH}_{4} \text { year }^{-1}\right)\end{array}$ \\
\hline \multicolumn{3}{|l|}{ Anthropogenic } \\
\hline $\begin{array}{l}\text { - Agriculture }{ }^{\mathrm{b}} \\
\text { - Industrial processes } \\
\text { - Residential and transport } \\
\text { - Waste } \\
\text { - Oil, coal and gas }\end{array}$ & $\begin{array}{l}\text { EDGAR-4.2 FT2010 } \\
\text { EDGAR-4.2 FT2010 } \\
\text { EDGAR-4.2 FT2010 } \\
\text { EDGAR-4.2 FT2010 } \\
\text { EDGAR-4.2 FT2010 }\end{array}$ & $\begin{array}{l}152.8 \\
0.3 \\
13.4 \\
61.5 \\
129.7\end{array}$ \\
\hline \multicolumn{3}{|l|}{ Natural } \\
\hline $\begin{array}{l}\text { - Wetlands } \\
\text { - Biomass burning } \\
\text { - Geological seeps } \\
\text { - Soils } \\
\text { - Wild animals } \\
\text { - Termites } \\
\text { - Ocean }\end{array}$ & $\begin{array}{l}\text { LPJ DGVM }^{\mathrm{g}} \\
\text { GFED-3.1 } \\
\text { Etiope et al. (2008) } \\
\text { Ridgwell et al. (1999) } \\
\text { Houweling et al. (1999) } \\
\text { Sanderson et al. (1996) } \\
\text { Lambert and Schmidt (1993) }\end{array}$ & $\begin{array}{l}175.0 \\
19.3 \\
55.3 \\
-37.9 \\
5.0 \\
19.3 \\
17.0\end{array}$ \\
\hline Total & & 610.0 \\
\hline
\end{tabular}

\subsubsection{Observations}

We used measurements of $\mathrm{CH}_{4}$ from approximately weekly samples in the National Oceanic and Atmospheric Administration Global Monitoring Division (NOAA GMD) Carbon Cycle and Greenhouse Gases (CCGG) network. These measurements are made using Gas Chromatographs fitted with Flame Ionization Detectors (GC-FID). In addition, we used data from a number of in situ measurement sites. These included in situ GC-FID instruments operated by the Umweltbundesamt (UBA), the Institute for Atmospheric Sciences and Climate (ISAC) and the Advanced Global Gases Experiment (AGAGE) as well as in situ Cavity Ring Down Spectrometers (CRDS) operated by EMPA and the Finnish Meteorological Institute (FMI). All measurements were reported as dry-air mole fractions in parts-per-billion (abbreviated as ppb) on the NOAA2004 calibration scale, except AGAGE data, which were reported on the Tohoku University scale but were converted to the NOAA2004 scale using a conversion factor of 1.0003 (see Table 3).

In situ measurements were assimilated as averages of the afternoon observations (12:00 to 18:00 LT) at low altitude sites and as averages of night-time observations at mountain sites (00:00 to 06:00 LT) and the corresponding FLEXPART SRRs were selected and averaged in the same way. Discrete measurements were assimilated as available and matched with the closest available 3-hourly SRR to the sampling time. The measurement error was defined as $5 \mathrm{ppb}$ based on the repeatability of the measurements and, in the case of the in situ data, the representation error was defined as the standard deviation of the afternoon observations.

\subsubsection{Prior fluxes and initial mixing ratios}

The prior flux was composed from estimates of anthropogenic and natural emissions from a number of different models and inventories (see Table 4 for details) and the total global source amounted to $610 \mathrm{Tg} \mathrm{CH}_{4}$ year ${ }^{-1}$. Methane fluxes were resolved monthly in the wetland, ocean, termite, wild animal, soil and biomass burning estimates, while the anthropogenic and geological flux estimates were only resolved annually. For the anthropogenic and biomass burning sources, the 2010 estimates were used, as no estimates were available for 2011. For the remaining natural sources, climatological estimates were used. All fluxes were used at a spatial resolution of $1.0^{\circ} \times 1.0^{\circ}$.

Prior flux error covariance matrix, $\mathbf{B}^{\text {flux }}$, was calculated as described in Sect. 2.5 using a spatial correlation length of $500 \mathrm{~km}, k_{\mathrm{S}}=500$, and a temporal correlation length of 90 days, $k_{\mathrm{T}}=90$. For the calculation of the flux errors we used a fraction of 0.5 of the maximum flux out of the cell of interest and the eight surrounding ones.

The background mixing ratios may be estimated either from the observations themselves or by coupling FLEXPART to a global model (see Sects. 2.1.2 and 2.3). For the latter method, FLEXPART calculates the sensitivity to the mixing ratio at the termination point of the virtual particles. These sensitivities were coupled to daily 3-D fields of 
$\mathrm{CH}_{4}$ mixing ratios from the atmospheric chemistry transport model, TM5, in order to calculate the initial mixing ratios. The TM5 model was run at $6.0^{\circ} \times 4.0^{\circ}$ horizontal resolution with 25 eta pressure levels using pre-optimized fields of $\mathrm{CH}_{4}$ fluxes (Bergamaschi et al., 2010). Atmospheric loss of $\mathrm{CH}_{4}$ by reaction with $\mathrm{OH}$ radicals is calculated in TM5 using monthly fields of $\mathrm{OH}$ concentration (Bergamaschi et al., 2005) resulting in mean atmospheric lifetime of $\mathrm{CH}_{4}$ of 10.1 years, which is close to the IPCC recommended value of 9.7 ( $\pm 20 \%$ ) years (Denman et al., 2007). The initial mixing ratios were added to the change in mixing ratios from fluxes outside the domain, together forming the background mixing ratio matrix, $\mathbf{M}_{\operatorname{cg}(M \times R)}$. The background was optimized at a resolution of $30^{\circ} \times 15^{\circ}$ (longitude by latitude) over the global domain (i.e. $R=144$ ). The uncertainty in the scalars of the background mixing ratio was set to $0.2 \%$ equivalent to approximately $4 \mathrm{ppb}$.

\subsection{Sensitivity tests}

We performed six inversions to test the sensitivity of the posterior fluxes and error reduction to the spatial correlation scale length ( $\mathrm{S} 1$ to $\mathrm{S} 3$ ), to the optimization of the background (S4), to the filtering and averaging of the observations (S5), as well as to the background estimation method (S6). The tests are summarized in Table 5.

\subsection{Results}

The inversions were run on a Linux Ubuntu machine with $62 \mathrm{~GB}$ memory. The maximum and mean memory usage was 18 and $6.4 \mathrm{~GB}$, respectively, and each inversion took approximately 1.8 days to complete.

Figure 3 shows the observed $\mathrm{CH}_{4}$ mixing ratios at in situ measurement sites compared with those simulated with the TM5 model and FLEXPART using the prior and posterior fluxes from test S1. At high-altitude sites, namely CMN, JFJ and SSL, the global model tends to underestimate the synoptic variability largely due to the coarse resolution. This can be quantified by the normalized standard deviation (NSD) (i.e. the SD of the model normalized by the SD of the observations), which for TM5 was 0.46, 0.81 and 0.71, compared with $0.81,0.75$ and 1.07 for FLEXPART, for the three sites respectively. On the other hand, TM5 overestimated the variability at MHD, a coastal site, with a NSD of 2.53 compared with 0.97 in FLEXPART. Again, this is likely to be due to the coarse resolution in TM5, which cannot accurately resolve the location of MHD and overestimates the influences of land fluxes at this site.

To examine the differences between the two methods of estimating the background mixing ratios, we compare the background determined in test S1 (model-based method) and test S6 (observation-based method). The results are shown in Fig. 4 at the in situ measurement sites. The two methods compare reasonably well with one another with the

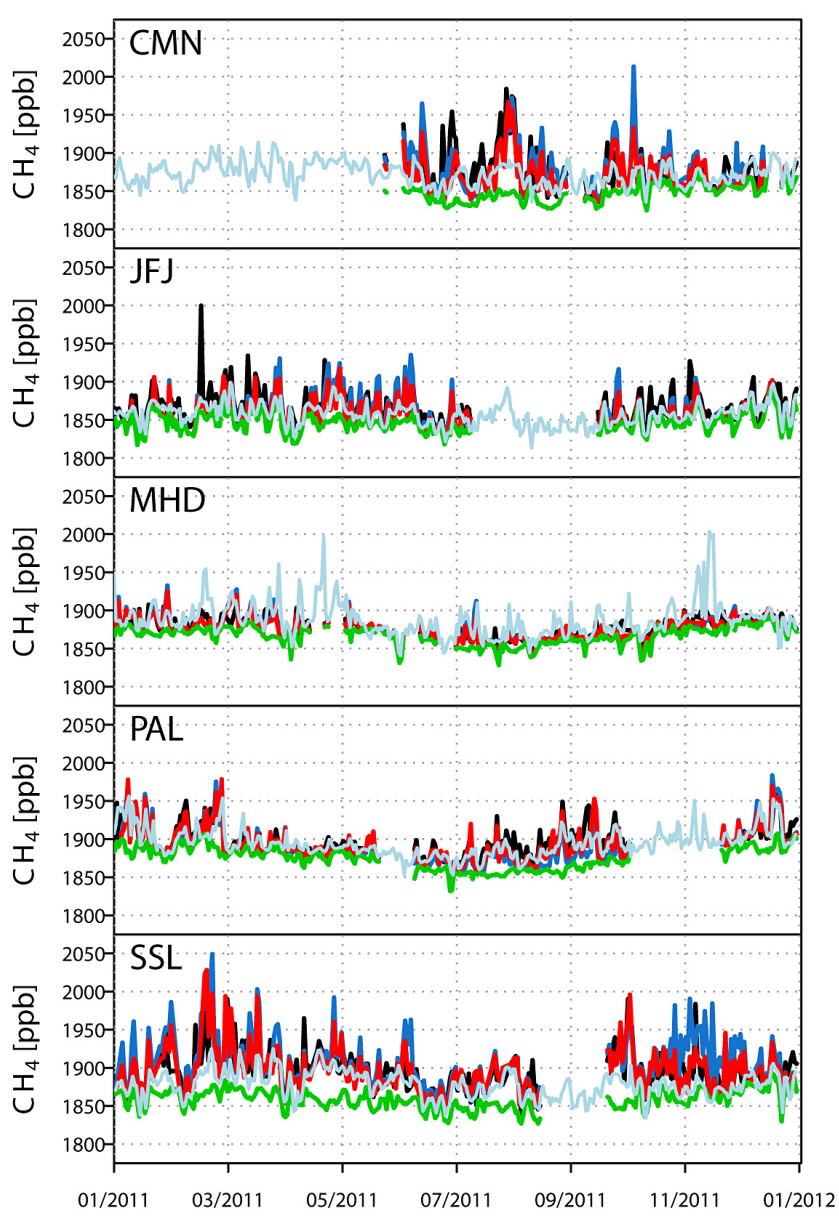

Figure 3. $\mathrm{CH}_{4}$ mole fractions (ppb) as observed (black) and simulated from the prior (blue) and posterior models (red). Also shown is the background $\mathrm{CH}_{4}$ mole fraction (green) and $\mathrm{CH}_{4}$ calculated by the TM5 Eulerian model (light blue).

mean difference between the two backgrounds being between -7 and $4 \mathrm{ppb}$ for the different sites. At MHD, however, observation-based background is considerably lower than the model-based one (a difference of $-11 \mathrm{ppb}$ ). This departure is caused by an overestimation of the prior contribution to the mixing ratio from fluxes inside the nested domain, and since this is subtracted from the observations that have been identified as being representative of the background, this leads to an overall too low background estimate at this site (see Sect. 2.1.2 for details).

The model performance at the measurement sites for test $\mathrm{S} 1$, a priori and a posteriori, is summarized in Table 6, which compares the correlation coefficient $(R)$, root mean square error (RMSE) and NSD of the simulated mixing ratios versus the observations. As expected, the mixing ratios a posteriori agree better with observations. To assess the assumptions made about the uncertainties and error correlation scales used in $\mathbf{B}$ and $\mathbf{R}$, we look at $\chi^{2}$, which has the value of the cost function at the optimum (equivalently the weighted sum of 
Table 5. Overview of the sensitivity tests.

\begin{tabular}{llll}
\hline Test ID & Spatial correlation & Observations & Background $^{\mathrm{a}}$ \\
\hline S1 & $500 \mathrm{~km}$ & afternoon/night only & model-based, not optimized \\
S2 & $300 \mathrm{~km}$ & afternoon/night only & model-based, not optimized \\
S3 & $200 \mathrm{~km}$ & afternoon/night only & model-based, not optimized \\
S4 & $500 \mathrm{~km}$ & afternoon/night only & model-based, optimized \\
S5 & $500 \mathrm{~km}$ & all $^{\mathrm{c}}$ & model-based, not optimized \\
S6 & $500 \mathrm{~km}$ & afternoon/night only & observation-based, not optimized \\
\hline
\end{tabular}

a The method of calculation and whether or not the background mixing ratios were optimized in the inversion. ${ }^{b}$ Low-altitude sites averaged afternoon data, high-altitude sites averaged night data. ${ }^{c}$ Averaged all data over $24 \mathrm{~h}$.

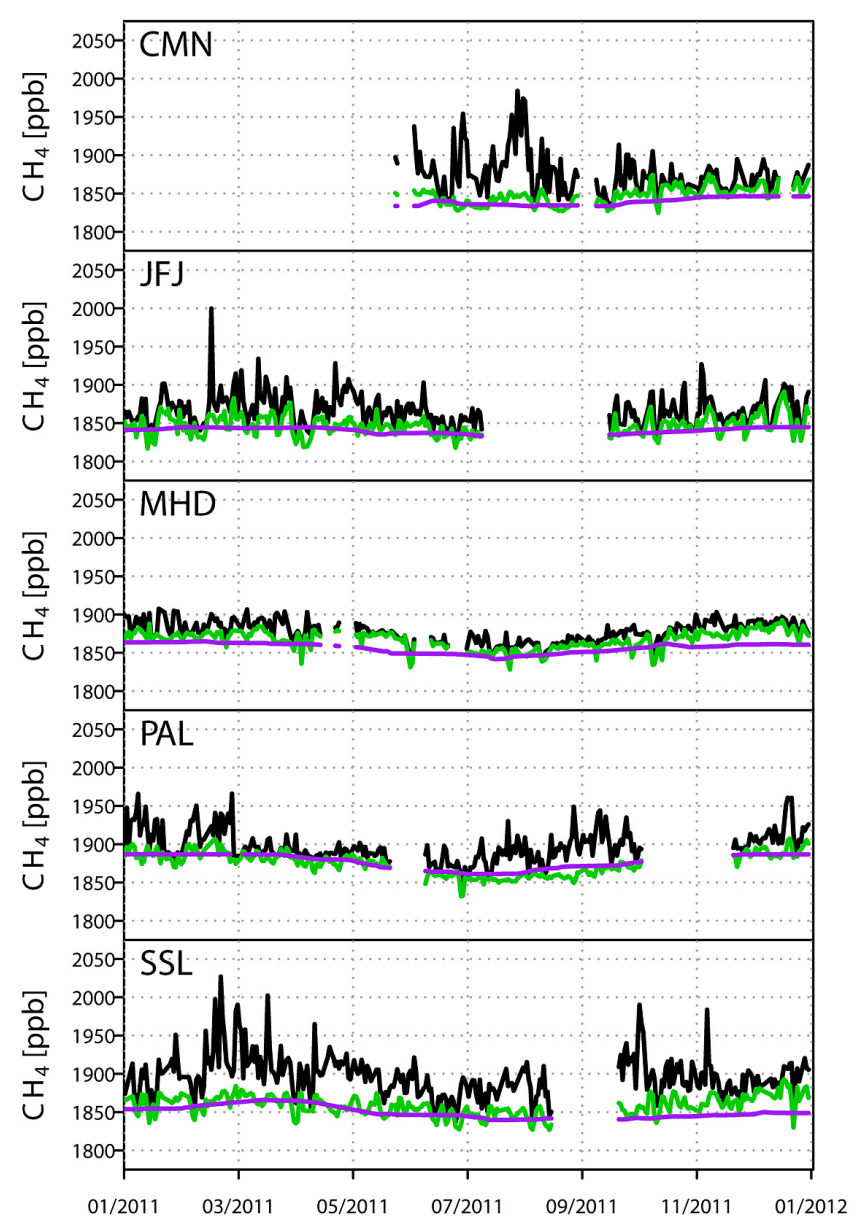

Figure 4. Comparison of the background $\mathrm{CH}_{4}$ mole fraction, calculated using the observation-based (purple) and the model-based methods (green), with the observed mixing ratio (black).

squares divided by the number of observations). Ideally, $\chi^{2}$ would be equal to 1 indicating that the posterior solution is within the limits of the prescribed uncertainties. In actual fact, the $\chi^{2}$ values are larger than $1 . \chi^{2}$ increased with increasing spatial correlation scale length with values of 2.24 , 2.56 and 2.97 with $k_{\mathrm{S}}$ of 200,300 and $500 \mathrm{~km}$, respectively, which is as expected since a longer correlation scale length
Table 6. Statistics of the simulated versus observed $\mathrm{CH}_{4}$ mixing ratios from test $\mathrm{S} 1$.

\begin{tabular}{|c|c|c|c|c|c|c|}
\hline \multirow[t]{2}{*}{ Site ID } & \multicolumn{3}{|l|}{ Prior } & \multicolumn{3}{|l|}{ Posterior } \\
\hline & NSD & $R$ & RMSE & NSD & $R$ & RMSE \\
\hline PAL & 0.99 & 0.69 & 16.2 & 1.04 & 0.82 & 11.9 \\
\hline ICE & 1.01 & 0.26 & 9.4 & 0.90 & 0.24 & 9.2 \\
\hline BAL & 1.14 & 0.66 & 16.6 & 0.96 & 0.72 & 13.7 \\
\hline MHD & 1.18 & 0.57 & 9.2 & 0.97 & 0.63 & 7.8 \\
\hline OXK* & - & - & 42.3 & - & - & 7.34 \\
\hline SSL & 1.21 & 0.52 & 28.2 & 1.07 & 0.71 & 19.3 \\
\hline HPB & 0.61 & 0.49 & 44.3 & 0.67 & 0.73 & 33.7 \\
\hline HUN & 0.54 & 0.69 & 47.5 & 0.96 & 0.88 & 27.1 \\
\hline JFJ & 1.04 & 0.30 & 21.4 & 0.75 & 0.33 & 20.3 \\
\hline BSC & 1.10 & 0.24 & 51.1 & 0.91 & 0.39 & 35.2 \\
\hline CMN & 1.00 & 0.56 & 26.4 & 0.81 & 0.68 & 21.6 \\
\hline CIB & 0.89 & 0.50 & 20.9 & 0.91 & 0.68 & 15.6 \\
\hline LMP & 2.02 & 0.34 & 35.0 & 1.68 & 0.45 & 24.0 \\
\hline
\end{tabular}

* Insufficient observations for calculating $R$ and NSD.

corresponds to fewer degrees of freedom. Using all observations, as in test $\mathrm{S} 5$, resulted in a $\chi^{2}$ of 2.05 , the lowest value, as this also resulted in larger SDs over the averaging interval (1 day) and, hence, larger uncertainties in the observation space.

The posterior fluxes and the flux increments (posterior minus prior fluxes) for the six sensitivity tests are shown in Fig. 5. The posterior fluxes and flux increments from tests $\mathrm{S} 1$ to S5 are quite similar. However, on close inspection there are a few notable differences. Decreasing the spatial correlation scale length from 500 to $200 \mathrm{~km}$ (tests S1 to S3) resulted in a more heterogeneous pattern of flux increments as the greater degrees of freedom allowed smaller spatial scales to be modified in the inversion - although, overall, the patterns of flux increments from all tests were consistent with lower emissions, relative to the prior, over France, Italy and the UK, and higher emissions over Austria, Hungary, and eastern Europe. When the background mixing ratios are also optimized (test S4) there is only a small change with respect to test S1; namely, in S4 the emissions are slightly lower over the United Kingdom, France and the Iberian Peninsular. Lower emissions are found as the background mixing 

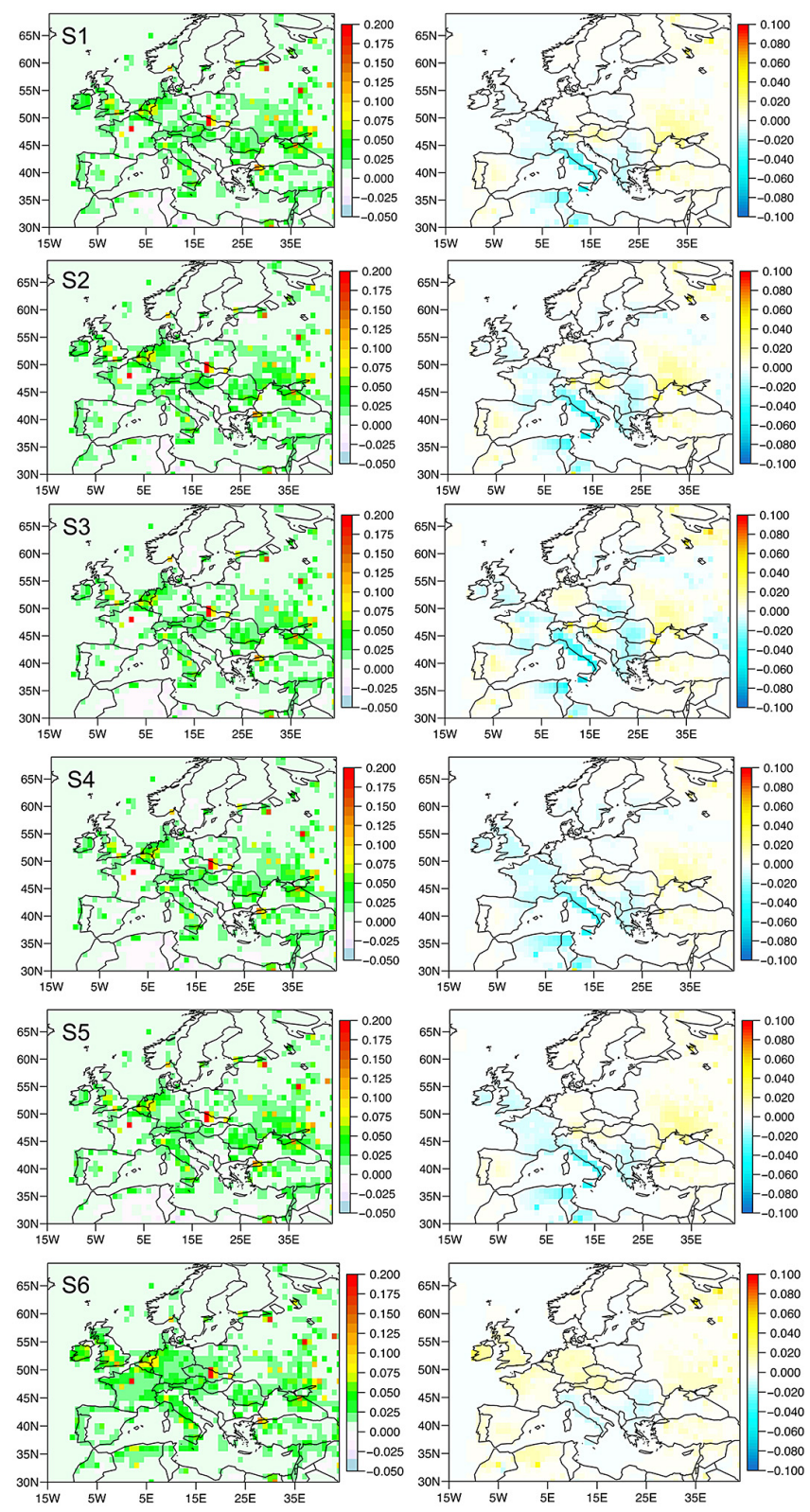

Figure 5. A posteriori fluxes of $\mathrm{CH}_{4}$ (left) and the flux increments (i.e. a posteriori - a priori fluxes) (right) for each of the sensitivity tests (in units of $\mathrm{g} \mathrm{CH}_{4} \mathrm{~m}^{-2} \mathrm{day}^{-1}$ ).

ratios have been increased (by approximately $0.2 \%$ ) to minimize the observation-model error and, hence, smaller increments were needed in the emissions. Furthermore, using all observations (test S5), compared to only afternoon ones at low-altitude sites and only night-time ones at high-altitude sites, made almost no difference to the posterior fluxes. Test S6, which used the observation-based approach for the background estimation, differed the most from the other tests. Notably, higher emissions, compared to the other inversions, were found in France, Germany, the Czech Republic and the UK and, correspondingly, the flux increments were more positive in these regions as well. This difference is a direct result of the lower background mixing ratios estimated at a number of sites with the observation-based method and highlights the challenge of obtaining robust background estimates.

Figure 6 shows the error reduction for the six sensitivity tests. The largest error reductions are found using $k_{\mathrm{S}}=$ $500 \mathrm{~km}$, i.e. in tests S1, S4, S5 and S6, for which the error reduction is almost identical. The error reductions in tests $\mathrm{S} 2$ and $\mathrm{S} 3$ are smaller and more limited to central Europe as compared to S1. Again, this is because increasing the correlation scale length results in fewer degrees of freedom for the inversion and effectively spreads the atmospheric information over a larger area.

Lastly, we compare the simulated mixing ratios using the a priori and a posteriori fluxes (from test S1) with observations at an independent site, i.e. one that was not included in the inversion, Puy de Dôme, France (PUY). Figure 7 shows the observed, prior, posterior and background mixing ratios at the timestamp of the observations at PUY. Both the prior and posterior mixing ratios overestimate the observed variability with NSD of 2.24 and 2.04, respectively. This is most probably owing to both the topography (the station is located on a volcanic cone, which represents a very abrupt change in topography) as well as the fact that there are significant emissions in the prior around the station. A likely explanation is that FLEXPART overestimates the BL height at PUY and thus overestimates the influence of local emissions on this site. Despite the model transport errors at this site, using the posterior fluxes improves the RMSE (23.1) and correlation coefficient (0.18) compared to the prior (26.4 and 0.16 , respectively).

\subsection{Discussion}

The results for the sensitivity tests S1 and S6, using the model and observation-based background mixing ratios, respectively, highlight the challenge of robustly identifying the background and the influence that this has on the optimized fluxes (see Fig. 5). There are different problems associated with each method which warrant further discussion here.

First, using an optimized global model (in our case study, the chemistry-transport model, TM5) to derive the prior background may lead to problems of circularity, i.e. if the background is included in the optimization and the same observations used to constrain the global model are also used in the Lagrangian inversion. If different sets of observations are used then this is not a problem. However, if there is overlap then the prior information (about the background) and the observations are no longer completely independent. The degree of overlap should, however, be small since the background calculated for the Lagrangian model is an extremely smoothed version of the global modelled mixing ratio fields, as the sensitivity to the background even for a single measurement is distributed over large parts of at least 

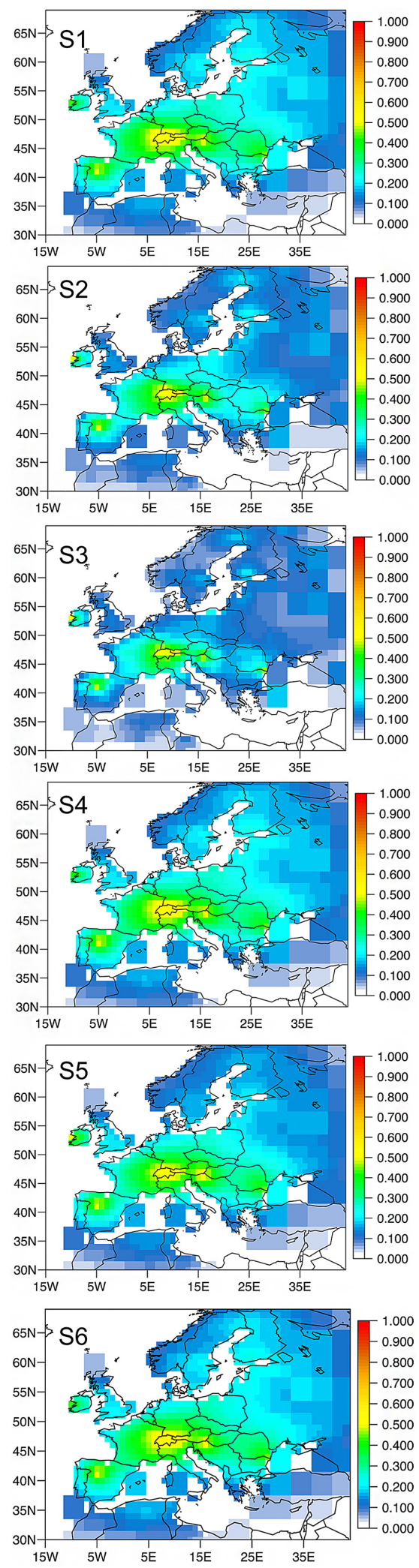

Figure 6. Error reduction for the $\mathrm{CH}_{4}$ fluxes for each of the sensitivity tests.

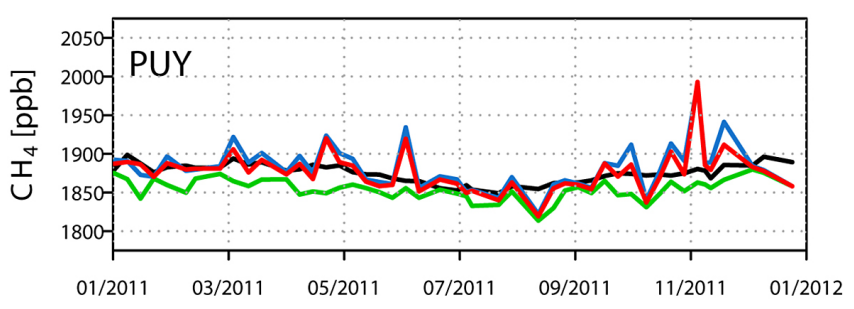

Figure 7. Comparison of the prior (blue), posterior (red) and background (green) simulated $\mathrm{CH}_{4}$ mixing ratios (ppb) with observations (black) at the independent site, PUY. Results are shown for test $\mathrm{S} 1$.

one hemisphere. Furthermore, in the global model, the background is constrained mainly by measurements from outside the region of interest. The degree of circularity is minimized even further if new observations are included in the Lagrangian inversion, which may also encompass assimilating observations from the same sites but at higher temporal resolution in the Lagrangian model if observations from no additional sites are available. In any case, the model-based background should be from a pre-optimized model or optimized in the Lagrangian inversion, as biases in the background will be propagated into biases in the optimized fluxes.

Second, using a filtering of the observations to derive the background will also lead to circularity, i.e. if the same observations are also used to optimize the background in the inversion, and this case should be avoided. When the observations are used to derive the background, biases only arise in the detection of the background signal. The background mixing ratio may fluctuate depending on the altitude and latitude of the air masses' origin. In addition, if the site is in an area of strong local fluxes, a background signal may not be detectable. Analysing the modelled back-trajectories in such cases may help determine whether candidate observations for the background calculation are likely to have been influenced by fluxes in the domain or not. Furthermore, the observationbased method for determining the background is not appropriate for species such as $\mathrm{CO}_{2}$, which have a strong diurnal cycle and thus no definable background.

We compare our posterior $\mathrm{CH}_{4}$ flux estimates with those derived from independent inverse models - specifically, with the results of a recent inverse model ensemble for $\mathrm{CH}_{4}$ fluxes over Europe from the NitroEurope project (Bergamaschi et al., 2014). This ensemble consisted of four independent models, including two Eulerian and two Lagrangian ones. Although the time period covered by the ensemble (2006 to 2007) differs from our study (2011), the fluxes of $\mathrm{CH}_{4}$ in Europe are thought to have been fairly stable between both periods and, hence, the differences are likely to represent differences in the model set-ups rather than only changes in the fluxes. Table 6 compares the prior and posterior emission totals from this study with those of Bergamaschi et al. (2014). Overall, the posterior fluxes from this study are within the 
Table 7. Comparison of $\mathrm{CH}_{4}$ emissions $\left(\mathrm{Tg} \mathrm{CH}_{4}\right.$ year $^{-1}$ ) from this study with the range of values from an inversion ensemble for 2006 and 2007 (Bergamaschi et al., 2014). The prior and posterior emissions are shown from test S1 and include the $1 \sigma$ SD prior and posterior uncertainties. NW Europe includes the UK, Ireland, BENELUX, France and Germany, and E Europe includes Hungary, Poland, the Czech Republic and Slovakia, according to the definitions in Bergamaschi et al. (2014).

\begin{tabular}{lccc}
\hline & Prior & Posterior & Bergamaschi et al. (2014) \\
\hline UK + Ireland & $2.66 \pm 0.84$ & $2.41 \pm 0.33$ & $2.32-4.57$ \\
BENELUX* & $1.18 \pm 0.80$ & $1.09 \pm 0.26$ & $1.44-2.29$ \\
France & $4.33 \pm 1.37$ & $3.14 \pm 0.42$ & $2.02-4.94$ \\
Germany & $2.22 \pm 1.16$ & $2.48 \pm 0.33$ & $2.35-3.51$ \\
NW Europe & $10.39 \pm 4.17$ & $9.12 \pm 1.34$ & $8.13-14.44$ \\
Hungary & $0.37 \pm 0.62$ & $0.50 \pm 0.17$ & $0.34-0.73$ \\
Poland & $2.81 \pm 1.05$ & $2.62 \pm 0.38$ & $1.84-2.87$ \\
Czech Republic + Slovakia & $1.18 \pm 0.94$ & $1.27 \pm 0.27$ & $1.12-1.63$ \\
E Europe & $4.36 \pm 2.61$ & $4.39 \pm 0.82$ & $3.59-4.90$ \\
NW + E Europe & $14.75 \pm 4.17$ & $13.51 \pm 2.16$ & $11.71-19.34$ \\
\hline
\end{tabular}

* BENELUX $=$ Belgium, The Netherlands and Luxembourg.

range from the ensemble, despite differences in the time period and the atmospheric observations used. There is only one exception, i.e. BENELUX, where our estimate is $24 \%$ lower than the lowest limit of the ensemble range. This may be due, at least in part, to real changes in emissions. However, it may also be due to differing distributions of the posterior emissions close to the boundaries of BENELUX with France and Germany, which considering the small area of BENELUX, may become important in the calculation of the total emission. Another contributing factor may also be that in the inversions in the Bergmaschi et al. (2014) study, the station, Cabauw $\left(52.0^{\circ} \mathrm{N}, 4.9^{\circ} \mathrm{E}\right)$, in the Netherlands, was included (whereas it was not included in our inversion), which likely also has a strong influence on the posterior fluxes in BENELUX.

\section{Summary and conclusions}

We have presented a new Bayesian inversion framework, FLEXINVERT, for the estimation of surface to atmosphere fluxes of atmospheric species. The framework is based on source-receptor relationships, which describe the relationship between changes in mixing ratio at a receptor "point" and changes in fluxes, calculated by the Lagrangian Particle Dispersion Model, FLEXPART. Fluxes may be optimized at any given temporal resolution and on a nested grid of variable spatial resolution. The variable grid is determined using the information of the integrated SRRs and has finer resolution where there is a strong observational constraint and coarser resolution where there is a weak constraint. In this framework, the background mixing ratio, i.e. the contribution to the mixing ratio at the receptors not accounted for by transport and fluxes inside the nested domain, is calculated by coupling FLEXPART to the output of a global Eulerian model (or alternatively, in the case that no such model output is available, it is calculated from the observations themselves). The background mixing ratios are also included in the optimization problem.

We demonstrated the performance of FLEXINVERT in a case study estimating $\mathrm{CH}_{4}$ fluxes over Europe in 2011. The posterior fluxes were found to compare well to the results from an independent inversion ensemble consisting of four different transport models and inversion frameworks. Although we have only presented an example for $\mathrm{CH}_{4}$, FLEXINVERT can be applied to any species for which atmospheric loss (if any) can be described as a linear process such as radioactive decay, dry and wet deposition, and oxidative chemistry. Furthermore, the framework can be used on continental, regional and even local scales with little or no modification. 
Appendix A: Optimization of the posterior fluxes to the fine-grid resolution

To optimize the posterior fluxes $\boldsymbol{f}_{\mathrm{vg}}^{\text {nest* }}$ on the variableresolution grid to the fine-resolution grid by applying Bayes' theorem (note that to simplify the notation we have used $f=f_{\mathrm{vg}}^{\text {nest* }}, f_{\mathrm{b}}=f^{\text {nest }}$, and $f^{*}=f^{\text {nest* }}$ i.e. the optimized fluxes on the fine grid),

$\rho\left(\boldsymbol{f}^{*} \mid \boldsymbol{f}\right)=\frac{\rho\left(\boldsymbol{f} \mid \boldsymbol{f}^{*}\right) \rho\left(\boldsymbol{f}^{*}\right)}{\rho(\boldsymbol{f})}$,

where $\rho\left(f^{*} \mid \boldsymbol{f}\right)$ is the posterior pdf that $\boldsymbol{f}^{*}$ lies in the interval $\left(\boldsymbol{f}^{*}, \boldsymbol{f}^{*}+d \boldsymbol{f}^{*}\right)$ when $\boldsymbol{f}$ (the "observation") has a given value. Assuming a Gaussian pdf and taking the natural logarithm we can express $\rho\left(\boldsymbol{f} \mid \boldsymbol{f}^{*}\right)$ as

$-2 \ln \rho\left(\boldsymbol{f} \mid \boldsymbol{f}^{*}\right)=\left(\boldsymbol{f}-\Gamma \boldsymbol{f}^{*}\right)^{\mathrm{T}} \mathbf{A}^{\text {flux-1 }}\left(\boldsymbol{f}-\Gamma \boldsymbol{f}^{*}\right)$,

(where $\mathbf{A}^{\text {flux }}$ is the posterior error covariance matrix and $\Gamma$ is the projection operator) and we can express $\rho\left(f^{*}\right)$ as

$-2 \ln \rho\left(\boldsymbol{f}^{*}\right)=\left(\boldsymbol{f}^{*}-\boldsymbol{f}_{b}\right)^{\mathrm{T}} \mathbf{B}^{\text {flux-1 }}\left(\boldsymbol{f}^{*}-\boldsymbol{f}_{b}\right)$,

(where $\mathbf{B}^{\text {flux }}$ is the prior error covariance matrix, on the fine grid) and by substituting Eqs. (2) and (3) into Eq. (1) we derive the expression for $\rho\left(\boldsymbol{f}^{*} \mid \boldsymbol{f}\right)$ :

$$
\begin{gathered}
-2 \ln \rho\left(\boldsymbol{f}^{*} \mid \boldsymbol{f}\right)=\left(\boldsymbol{f}^{*}-\boldsymbol{f}_{b}\right)^{\mathrm{T}} \mathbf{B}^{\text {flux-1 }}\left(\boldsymbol{f}^{*}-\boldsymbol{f}_{b}\right) \\
+\left(\boldsymbol{f}-\Gamma \boldsymbol{f}^{*}\right)^{\mathrm{T}} \mathbf{A}^{\text {flux-1 }}\left(\boldsymbol{f}-\Gamma \boldsymbol{f}^{*}\right) .
\end{gathered}
$$

The cost function can be thus be defined as

$$
\begin{aligned}
J\left(f^{*}\right)= & \frac{1}{2}\left(\boldsymbol{f}^{*}-\boldsymbol{f}_{b}\right)^{\mathrm{T}} \mathbf{B}^{\text {flux-1 }}\left(\boldsymbol{f}^{*}-\boldsymbol{f}_{b}\right) \\
& +\frac{1}{2}\left(\boldsymbol{f}-\Gamma \boldsymbol{f}^{*}\right)^{\mathrm{T}} \mathbf{A}^{\text {flux-1}}\left(\boldsymbol{f}-\Gamma \boldsymbol{f}^{*}\right)
\end{aligned}
$$

and the first derivative as

$$
\begin{aligned}
J^{\prime}\left(f^{*}\right)= & \mathbf{B}^{\text {flux-1 }}\left(\boldsymbol{f}^{*}-\boldsymbol{f}_{b}\right) \\
& -\Gamma^{\mathrm{T}} \mathbf{A}^{\text {flux-1 }}\left(\boldsymbol{f}-\Gamma \boldsymbol{f}^{*}\right) .
\end{aligned}
$$

Thus we can derive the expression for $\boldsymbol{x}^{*}$ at the minimum:

$$
\begin{aligned}
\boldsymbol{f}^{*}= & \boldsymbol{f}_{b} \\
& +\mathbf{B}_{\text {naw }}^{\text {flux }} \Gamma_{\text {unit }}^{\mathrm{T}}\left(\Gamma \mathbf{B}^{\text {flux }} \Gamma^{\mathrm{T}}+\mathbf{A}^{\text {flux }}\right)^{-1}\left(\boldsymbol{f}-\Gamma \boldsymbol{f}_{b}\right) .
\end{aligned}
$$

\section{Appendix B: Description of the software}

\section{B1 General description}

The code corresponding to the inversion framework described in this paper is called FLEXINVERT and is available from the website: http://flexinvert.nilu.no under a GNU General Public License. FLEXINVERT is coded in Fortran90 and has been tested with the gfortran compiler and the Linux Ubuntu operating system and a makefile for gfortran is included. To run FLEXINVERT, the LAPACK and NetCDF libraries for Fortran must be installed. The current version of FLEXINVERT can be run directly with output from FLEXPART 9.2.

\section{B2 Input data}

FLEXINVERT uses two definition files, the first specifies the paths, filenames, and other file-related information (files.def), and the second specifies the settings for each inversion run, such as the domain, dates, and uncertainties (control.def).

\section{- FLEXPART files}

FLEXINVERT looks for FLEXPART output files for each receptor in directories with the following naming convention: /STATION/YYYYMM/ where STATION is the name of the receptor and must be the same as that given in the station list file and in the prefix of the observation files. The FLEXPART files required are: header, grid_time (and grid_initial when computing the background using global model output). It is important to note that if the full 3D SRR fields are saved in the grid_time files, the reading of these files becomes considerably slower. Therefore, it is recommended to save only the surface layer of the SRR fields in the grid_time files. However, if the grid_initial files are used, these need all layers. (An option for this grid_time and grid_initial was added into FLEXPART 9.2). Also, note that FLEXINVERT expects the stochastic errors to be written to the grid_time files. If these are not written then a minor modification is required in readgrid. $f 90$.

- Station list file

This file specifies the receptors (where there are observations) to include in the inversion. The default file has the following format: receptor name, latitude, longitude, altitude, observation type (either CM for continuous or FM for flask measurement) and a character string of up to 55 characters describing the receptor. However, only the receptor name and type are actually used in the inversion:

ID LAT LON ALT TYP STATIONNAME

STATION XX.XX XXX.XX XXXX XX Station Name, Country

\section{- Observations}

The sub-routine readobs. 190 reads the observations from a separate ASCII file for each receptor. Again, FLEXINVERT looks for the file prefix STATION. 
The files contain six columns: year, month, day, hour, minute, mixing ratio - and optionally the measurement error estimate.

- Prior fluxes

The sub-routine reademissions.f90 reads the prior fluxes (or equivalently prior emissions) from a NetCDF file containing a 3-D floating variable for the fluxes with dimensions time, latitude and longitude, and the corresponding dimension variables. The name of the floating point variable is specified in files. def by the variable emisname.

- Landcover file

FLEXINVERT uses high-resolution landcover data to specify areas of water when determining the variable resolution grid. By default, FLEXINVERT uses the IGBP data, which is included in the tar archive.

- Land-sea mask file

A land-sea mask file is used in FLEXINVERT to determine which grid cells are on land/ocean when calculating the covariance matrix. The default land-sea mask is at $0.125^{\circ} \times 0.125^{\circ}$ resolution and is converted to the needed resolution automatically.

\section{- 3-D concentration fields}

For the calculation of the initial mixing ratios from a global model, its 3-D concentration fields are needed. FLEXINVERT includes routines for reading the output of the models LMDZ4 and TM5 in NetCDF format, which can be used as templates for reading data from other models.

\section{B3 Output data}

At the end of an inversion run, FLEXINVERT writes the output into the following files:

- obsread.txt

ASCII file containing the observed, prior, posterior and background mixing ratios at the same timestamp as the observations. Note that if the background is not optimized, then the observed, prior and posterior mixing ratios are the difference from the background and the values in the column BGND_POST are zero. The obsread.txt file has the following format:

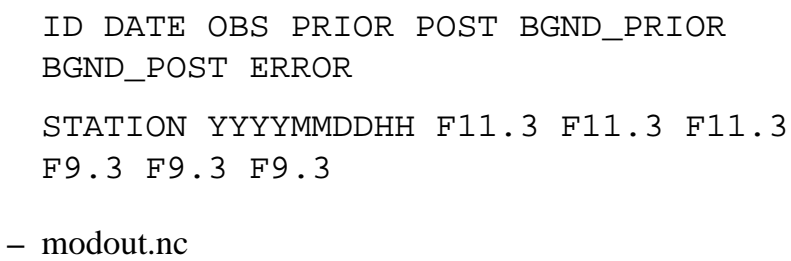

- modout.nc

NetCDF file containing floating point variables for the prior and posterior mixing ratios (ypri and ypos, respectively) as well as the prior and posterior background mixing ratios (bgpri and bgpos, respectively). These mixing ratios are computed using the fluxes at the finest resolution and at the timestamp of the FLEXPART trajectories. The variables have dimension of time and receptor.

- analysis.nc

NetCDF file containing floating point variables for the prior and posterior fluxes (emis_prior and emis_post, respectively) as well as the prior and posterior flux errors (error_prior and error_post, respectively). The variables are in dimensions of longitude, latitude and time and have units of $\mathrm{kg} \mathrm{m}^{-2} \mathrm{~s}^{-1}$.

- covb.nc

NetCDF file containing a floating point variable of the prior error covariance matrix (covb) and units $\left(\mathrm{kg} \mathrm{m}^{-3} \mathrm{~s}^{-1}\right)^{2}$. Note that the errors are scaled by the numerical scaling factor defined in mod_var.f 90 .

- cova.nc

As for covb.nc but containing the posterior error covariance matrix (cova).

- covr.nc

NetCDF file containing a floating point variable of the observation error covariance matrix (covr) with units of mixing ratio squared (e.g. $\mathrm{ppb}^{2}$ ).

- nbox_xy.nc

NetCDF file containing a floating point variable of the mapping of the fine to the variable resolution grid with dimensions of the number of longitudinal by latitudinal grid cells.

For testing purposes, the following files are also written but in most cases will not be required:

- gain.nc

NetCDF file containing the Gain matrix, $\mathbf{B H}^{\mathrm{T}}\left(\mathbf{H B H}^{\mathrm{T}}+\right.$ $\mathbf{R})^{-1}\left(\boldsymbol{y}-\mathbf{H} \boldsymbol{x}_{\mathrm{b}}\right)$.

- covbfin.nc

NetCDF file of the prior error covariance matrix on the fine grid (covbfin) with units $\left(\mathrm{kg} \mathrm{m}^{-3} \mathrm{~s}^{-1}\right)^{2}$. Note that the errors are scaled by the numerical scaling factor.

- covbfinaw.nc

As for covbfin.nc but containing the area-weighted errors (covbfinaw). 


\section{- covagg.nc}

NetCDF file of the aggregation errors in units of mixing ratio squared (e.g. $\mathrm{ppb}^{2}$ ).

- grid_operator.nc

NetCDF file of the projection operator, $\Gamma$, from the fine to the variable grid.

- grid_coarse.nc

NetCDF file of the projection operator, $\Gamma_{\mathrm{cg}}$, from the fine to the coarse grid.

- emisflex.nc

NetCDF file of the prior emissions converted to the FLEXPART (i.e. fine) grid in units of $\mathrm{kg} \mathrm{m}^{-3} \mathrm{~s}^{-1}$.

- knest_finobs.nc

NetCDF file of the transport operator, $\mathbf{H}^{\text {nest }}$, for the fine grid and averaged to the observation averaging interval.

- knest_obs.nc

NetCDF file of the transport operator, $\mathbf{H}^{\text {nest }}$, for the variable grid and averaged to the observation averaging interval.

- knest_trim.nc

NetCDF file of the transport operator, $\mathbf{H}^{\text {nest }}$, for the variable grid with rows matching observations.

- kout_obs.nc

NetCDF file of the initial mixing ratio contributions, $\mathbf{H}^{\text {out }} \boldsymbol{y}^{\text {out }}$, for the coarse grid and averaged to the observation averaging interval.

- immr.nc

NetCDF file of the 3-D initial mixing ratios from the global model (for option bgmethod $=2$ only) interpolated to the FLEXPART resolution (first time step only).

- area_box.txt

ASCII file containing a vector of the variable grid cell areas $\left(\mathrm{m}^{2}\right)$.

- prior.txt

ASCII file containing the prior state vector, $\boldsymbol{x}_{\mathrm{b}}$.

- posterior.txt

ASCII file containing the posterior state vector, $\boldsymbol{x}$.

- bgscalars.txt

ASCII file containing the prior and posterior scalars of the background mixing ratios and their errors with the format:

PRIOR POST PRIOR_ERROR POST_ERROR

F6. 4 F6. 4 F6. 4 F6. 4 F6. 4

\section{Appendix C: Applying inequality constraints}

After running FLEXINVERT, a separate code may be run to apply inequality constraints. The inequality constraint code is similarly written in Fortran90 and has been tested with the gfortran compiler and the Linux operating system. To run the code, the LAPACK and NetCDF libraries for Fortran must be installed. This code is available from the website: http://flexinvert.nilu.no. 
Acknowledgements. We thank all those who provided $\mathrm{CH}_{4}$ measurements for use in the case study, namely, E. Dlugokencky (NOAA), M. Steinbacher (EMPA), J. Arduini (ISAC), K. Uhse (UBA), J. Hatakka (FMI) and S. O'Doherty (University of Bristol). We also thank P. Bergamaschi for the use of the TM5 model output, as well as for providing the results from the inversion ensemble of $\mathrm{CH}_{4}$ fluxes in Europe. In addition, we thank I. Pisso for his help improving this paper. This work was financed by the Norwegian Research Council projects SOGG-EA and GHG-Nor.

Edited by: A. Archibald

\section{References}

Belward, A. S., Estes, J. E., and Kline, K. D.: The IGBP-DIS global 1-km land-cover data set DISCover: A project overview, Photogram. Eng. Remote Sens., 65, 1013-1020, 1999.

Bergamaschi, P., Krol, M., Dentener, F., Vermeulen, A., Meinhardt, F., Graul, R., Ramonet, M., Peters, W., and Dlugokencky, E. J.: Inverse modelling of national and European $\mathrm{CH}_{4}$ emissions using the atmospheric zoom model TM5, Atmos. Chem. Phys., 5, 2431-2460, doi:10.5194/acp-5-2431-2005, 2005.

Bergamaschi, P., Krol, M., Meirink, J. F., Dentener, F., Segers, A., van Aardenne, J., Monni, S., Vermeulen, A. T., Schmidt, M., Ramonet, M., Yver, C., Meinhardt, F., Nisbet, E. G., Fisher, R. E., O'Doherty, S., and Dlugokencky, E. J.: Inverse modeling of European $\mathrm{CH}_{4}$ emissions 2001-2006, J. Geophys. Res., 115, D22309, doi:10.1029/2010jd014180, 2010.

Bergamaschi, P., Corazza, M., Karstens, U., Athanassiadou, M., Thompson, R. L., Pison, I., Manning, A. J., Bousquet, P., Segers, A., Vermeulen, A. T., Janssens-Maenhout, G., Schmidt, M., Ramonet, M., Meinhardt, F., Aalto, T., Haszpra, L., Moncrieff, J., Popa, M. E., Lowry, D., Steinbacher, M., Jordan, A., O'Doherty, S., Piacentino, S., and Dlugokencky, E.: Top-down estimates of European $\mathrm{CH}_{4}$ and $\mathrm{N}_{2} \mathrm{O}$ emissions based on four different inverse models, Atmos. Chem. Phys. Discuss., 14, 15683-15734, doi:10.5194/acpd-14-15683-2014, 2014.

Brunner, D., Henne, S., Keller, C. A., Reimann, S., Vollmer, M. K., O'Doherty, S., and Maione, M.: An extended Kalman-filter for regional scale inverse emission estimation, Atmos. Chem. Phys., 12, 3455-3478, doi:10.5194/acp-12-3455-2012, 2012.

Chevallier, F., Fisher, M., Peylin, P., Serrar, S., Bousquet, P., Bréon, F. M., Chédin, A., and Ciais, P.: Inferring $\mathrm{CO}_{2}$ sources and sinks from satellite observations: Method and application to TOVS data, J. Geophys. Res., 110, D24309, doi:10.1029/2005jd006390, 2005.

Denman, K. L., Brasseur, G. P., Chidthaisong, A., Ciais, P., Cox, P. M., Dickinson, R. E., Hauglustaine, D., Heinze, C., Holland, E., Jacob, D., Lohmann, U., Ramachandran, S., da Silva Dias, P. L., Wofsy, S. C., and Zhang, X.: Couplings Between Changes in the Climate System and Biogeochemistry, in Climate Change 2007: The Physical Science Basis. Contribution of Working Group I to the Fourth Assessment Report of the Intergovernmental Panel on Climate Change, edited by: Solomon, S. D., Qin, D., Manning, M., Chen, Z., Marquis, M., Averyt, K. B., Tignor, M., and Miller, H. L., 499-587, Cambridge University Press, Cambridge, 2007.

Enting, I. G.: Inverse Problems in Atmospheric Constituent Transport, Cambridge University Press, Cambridge, New York, 2002.
Etiope, G., Lassey, K. R., Klusman, R. W., and Boschi, E.: Reappraisal of the fossil methane budget and related emission from geologic sources, Geophys. Res. Lett., 35, L09307, doi:10.1029/2008g1033623, 2008.

Flesch, T. K., Wilson, J. D., and Yee, E.: Backward-time Lagrangian stochastic dispersion models and their application to estimate gaseous emissions, J. App. Meteor., 34, 1320-1332, 1995.

Fung, I., John, J., Lerner, J., Matthews, E., Prather, M., Steele, L. P., and Fraser, P. J.: Three-dimensional model synthesis of the global methane cycle, J. Geophys. Res., 96, 13033-13065, doi:10.1029/91JD01247, 1991.

Gerbig, C., Lin, J. C., Wofsy, S. C., Daube, B. C., Andrews, A. E., Stephens, B. B., Bakwin, P. S., and Grainger, C. A.: Toward constraining regional-scale fluxes of $\mathrm{CO}_{2}$ with atmospheric observations over a continent: 2 . Analysis of COBRA data using a receptor-oriented framework, J. Geophys. Res., 108, 4757, doi:10.1029/2003jd003770, 2003.

Giostra, U., Furlani, F., Arduini, J., Cava, D., Manning, J., O'Doherty, J., Reimann, S., and Maione, M.: The determination of a "regional" atmospheric background mixing ratio for anthropogenic greenhouse gases: A comparison of two independent methods, Atmos. Environ., 45, 7396-7405, doi:10.1016/j.atmosenv.2011.06.076, 2011.

Houweling, S., Kaminski, T., Dentener, F., Lelieveld, J., and Heimann, M.: Inverse modeling of methane sources and sinks using the adjoint of a global transport model, J. Geophys. Res., 104, 26137-26160, doi:10.1029/1999jd900428, 1999.

Kaminski, T., Heimann, M. and Giering, R.: A coarse grid threedimensional global inverse model of the atmospheric transport: 2. Inversion of the transport of $\mathrm{CO}_{2}$ in the 1980s, J. Geophys Res., 104, 18555-18581, doi:10.1029/1999JD900146, 1999.

Kaminski, T., Rayner, P. J., Heimann, M., and Enting, I. G.: On aggregation errors in atmospheric transport inversions, J. Geophys. Res, 106, 4703-4715, doi:10.1029/2000JD900581, 2001.

Keller, C. A., Hill, M., Vollmer, M. K., Henne, S., Brunner, D., Reimann, S., O’Doherty, S., Arduini, J., Maione, M., Ferenczi, Z., Haszpra, L., Manning, A. J., and Peter, T.: European emissions of halogenated greenhouse gases inferred from atmospheric measurements, Environ. Sci. Technol., 46, 217-225, doi:10.1021/es202453j, 2012.

Koyama, Y., Maksyutov, S., Mukai, H., Thoning, K., and Tans, P.: Simulation of variability in atmospheric carbon dioxide using a global coupled Eulerian - Lagrangian transport model, Geosci. Model Dev., 4, 317-324, doi:10.5194/gmd-4-317-2011, 2011.

Lambert, G. and Schmidt, S.: Reevaluation of the oceanic flux of methane: Uncertainties and long term variations, Chemosphere, 26, 579-589, doi:10.1016/0045-6535(93)90443-9, 1993.

Lauvaux, T., Gioli, B., Sarrat, C., Rayner, P. J., Ciais, P., Chevallier, F., Noilhan, J., Miglietta, F., Brunet, Y., Ceschia, E., Dolman, H., Elbers, J. A., Gerbig, C., Hutjes, R., Jarosz, N., Legain, D., and Uliasz, M.: Bridging the gap between atmospheric concentrations and local ecosystem measurements, Geophys. Res. Lett., 36, L19809, doi:10.1029/2009g1039574, 2009.

Manning, A. J., Ryall, D. B., and Derwent, R. G.: Estimating European emissions of ozone-depleting and greenhouse gases using observations and a modeling back-attribution technique, J. Geophys. Res., 108, 4405, doi:10.1029/2002JD002312, 2003.

Manning, A. J., O’Doherty, S., Jones, A. R., Simmonds, P. G., and Derwent, R. G.: Estimating UK methane and nitrous oxide emis- 
sions from 1990 to 2007 using an inversion modeling approach, J. Geophys. Res., 116, D02305, doi:10.1029/2010jd014763, 2011.

Rayner, P. J., Enting, I. G., Francey, R. J., and Langenfelds, R.: Reconstructing the recent carbon cycle from atmospheric $\mathrm{CO}_{2}, \delta^{13} \mathrm{C}$ and $\mathrm{O}_{2} / \mathrm{N}_{2}$ observations, Tellus $\mathrm{B}, 51,213-232$, doi:10.1034/j.1600-0889.1999.t01-1-00008.x, 1999.

Ridgwell, A. J., Marshall, S. J., and Gregson, K.: Consumption of atmospheric methane by soils: A process-based model, Global Biogeochem. Cy., 13, 59-70, doi:10.1029/1998gb900004, 1999.

Rigby, M., Manning, A. J., and Prinn, R. G.: Inversion of long-lived trace gas emissions using combined Eulerian and Lagrangian chemical transport models, Atmos. Chem. Phys., 11, 9887-9898, doi:10.5194/acp-11-9887-2011, 2011.

Rödenbeck, C., Gerbig, C., Trusilova, K., and Heimann, M.: A twostep scheme for high-resolution regional atmospheric trace gas inversions based on independent models, Atmos. Chem. Phys., 9, 5331-5342, doi:10.5194/acp-9-5331-2009, 2009.

Ruckstuhl, A. F., Jacobson, M. P., Field, R. W., and Dodd, J. A.: Baseline subtraction using robust local regression estimation, J. Quant. Spectr. Radiat. Transf., 68, 179-193, doi:10.1016/S00224073(00)00021-2, 2001.

Sanderson, M. G.: Biomass of termites and their emissions of methane and carbon dioxide: A global database, Global Biogeochem. Cy., 10, 543-557, doi:10.1029/96gb01893, 1996.

Seibert, P. and Frank, A.: Source-receptor matrix calculation with a Lagrangian particle dispersion model in backward mode, Atmos. Chem. Phys., 4, 51-63, doi:10.5194/acp-4-51-2004, 2004.

Stohl, A., Hittenberger, M., and Wotawa, G.: Validation of the Lagrangian particle dispersion model FLEXPART against largescale tracer experiment data, Atmos. Environ., 32, 4245-4264, 1998.

Stohl, A., Forster, C., Eckhardt, S., Spichtinger, N., Huntrieser, H., Heland, J., Schlager, H., Wilhelm, S., Arnold, F., and Cooper, O.: A backward modeling study of intercontinental pollution transport using aircraft measurements, J. Geophys. Res.-Atmos., 108, 4370, doi:10.1029/2002JD002862, 2003.
Stohl, A., Forster, C., Frank, A., Seibert, P., and Wotawa, G.: Technical note: The Lagrangian particle dispersion model FLEXPART version 6.2, Atmos. Chem. Phys., 5, 2461-2474, doi:10.5194/acp-5-2461-2005, 2005.

Stohl, A., Seibert, P., Arduini, J., Eckhardt, S., Fraser, P., Greally, B. R., Lunder, C., Maione, M., Mühle, J., O’Doherty, S., Prinn, R. G., Reimann, S., Saito, T., Schmidbauer, N., Simmonds, P. G., Vollmer, M. K., Weiss, R. F., and Yokouchi, Y.: An analytical inversion method for determining regional and global emissions of greenhouse gases: Sensitivity studies and application to halocarbons, Atmos. Chem. Phys., 9, 1597-1620, doi:10.5194/acp-91597-2009, 2009.

Stohl, A., Kim, J., Li, S., O’Doherty, S., Mühle, J., Salameh, P. K., Saito, T., Vollmer, M. K., Wan, D., Weiss, R. F., Yao, B., Yokouchi, Y., and Zhou, L. X.: Hydrochlorofluorocarbon and hydrofluorocarbon emissions in East Asia determined by inverse modeling, Atmos. Chem. Phys., 10, 35453560, doi:10.5194/acp-10-3545-2010, 2010.

Tans, P. P., Fung, I. Y., and Takahashi, T.: Observational constraints on the global atmospheric $\mathrm{CO}_{2}$ budget, Science, 247, 14311438, 1990.

Tarantola, A.: Inverse problem theory and methods for model parameter estimation, Society for Industrial and Applied Mathematics, Philadelphia, 358 pp., 2005.

Thacker, W. C.: Data assimilation with inequality constraints, Ocean Model., 16, 264-276, doi:10.1016/j.ocemod.2006.11.001, 2007.

Thompson, R. L., Gerbig, C., and Rödenbeck, C.: A Bayesian inversion estimate of $\mathrm{N}_{2} \mathrm{O}$ emissions for western and central Europe and the assessment of aggregation errors, Atmos. Chem. Phys., 11, 3443-3458, doi:10.5194/acp-11-3443-2011, 2011.

Trampert, J. and Snieder, R.: Model estimations biased by truncated expansions: possible artefacts in seismic tomography, Science, 271, 1257-1260, 1996.

Wu, L., Bocquet, M., Lauvaux, T., Chevallier, F., Rayner, P., and Davis, K.: Optimal representation of source-sink fluxes for mesoscale carbon dioxide inversion with synthetic data, J. Geophys. Res.-Atmos., 116, D21304, doi:10.1029/2011jd016198, 2011. 